\title{
Study on Cross Compatibility of Intergeneric Hybridization between Carica with Vasconcellea Species
}

\author{
Deepa U. Pujar*, C. Vasugi ${ }^{1}$, D. Adiga ${ }^{2}$, M.K. Honnabyraiah ${ }^{3}$, \\ H.S. Vageeshbabu ${ }^{1}$, J. Jayappa ${ }^{3}$ and Kanupriya ${ }^{1}$ \\ ${ }^{1} I C A R-I I H R$, Bengaluru, India \\ ${ }^{2}$ ICAR-DCR, Puttur, India \\ ${ }^{3} \mathrm{COH}$, Bengaluru, India
}

*Corresponding author

\section{A B S T R A C T}

\section{Keywords}

Papaya, intergeneric hybridization, Vasconcellea, compatibility

Article Info

Accepted: 12 March 2019 Available Online: 10 April 2019
Intergeneric hybridization was carried out to know the cross compatibility between papaya varieties (Arka Prabhath, Arka Surya and Red Lady) with Vasconcellea species (V. cauliflora, V. cundinamarcensis and V. parviflora). Arka Prabhath was found to be a good combiner with all the three wild species ( $V$. cauliflora, $V$. cundinamarcensis and $V$. parviflora) which has recorded good fruit set $(85.42,78.13$ and $84.38 \%$ respectively), fruit retention $(98.96,83.33$ and $73.96 \%$ respectively) and more mean number of fertile seeds per fruit $(63.99,70.82$ and 28.14 respectively). Among male parents, Vasconcellea. cauliflora was found to be good combiner with all three female parents (Arka Prabhath, Arka Surya and Red Lady) which has resulted better fruit set $(85.42,85.42$ and $76.04 \%$ respectively), fruit retention $(98.96,19.79,43.75 \%$ respectively) and more mean number of fertile seeds per fruit $(63.99,11.00$ and 4.50 respectively). Hence Arka Prabhath with $V$. cauliflora cross combination was found to be good for intergeneric hybridization which has resulted in more mean number of fertile seeds (heterotic combinations) which ultimately helps in generating more number of intergeneric population for screening of papaya ring spot virus (PRSV).

\section{Introduction}

Papaya with its botanical name as Carica papaya L. belongs to the family Caricaceae, is one amongst the economically vital fruit crops of both subtropical and tropical zones of the world. It is basically a tropical fruit crop and believed to have originated from Mexico to Panama (Nakasone and Paull, 1998).
Papaya is a polygamous species with three basic sex forms as male (staminate), female (pistillate) and hermaphrodite forms of inflorescence (Storey, 1938; Singh, 1964). It is a diploid species with a small genome of $372 \mathrm{Mbp} / 1 \mathrm{C}$ (Arumuganathan and Earle, 1991) and nine pairs of chromosomes (Bennett and Leitch, 2005). The fruit of papaya has high nutritive and medicinal value 
(Azad et al., 2012) especially vitamin A (2020 IU/100g). The proteolytic enzyme 'papain' obtained from raw fruits of papaya is used for meat tenderization, wool preshrinking, chewing gum preparation, removal of gum from natural silk and in preparation of many cosmetics. Papaya has been successfully cultivated in India, USA, Mexico, Peru, Brazil, Jamaica, Nigeria, China, Taiwan, Indonesia, Phillippines and Thailand.

According to recent past data, papaya cultivation with respect area has increasing tend but with respect to production is stagnant or the consequent production has not observed. This might be due to several factors of biotic such as diseases caused by bacterial, fungal, viral and phytoplasma and many pest attack and abiotic factors such as drastic variation in temperature, humidity, soil moisture and irregular rains.

In recent years, throughout the world, papaya ringspot virus (PRSV) type P Litz, (1984) is the most destructive disease of papaya which is a perfect poty virus from poty viridae (Shukla et al., 1994). PRSV is grouped into two types as PRSV-P and PRSV-W. The infection level is differing for both types as PRSV-P infects both papaya and cucurbits where as PRSV cannot infect papaya (Gonsalves, 1998).The incidence of PRSV has been reported to be more than 90 per cent in India (Hussain and Varma, 1994; Chandra and Samuel, 1999) and rendering papaya orchards economically unviable. Almost all cultivated varieties belong to the genus Carica are highly susceptible to PRSV. However, much effort is being spent to introduce resistant genes from wild species even though the resistance appears to be variable and depend on the geographical origin of the virus environmental conditions (Gonsalves et al., 2004). Resistance against PRSV was identified in genus Vasconcellea species viz., Vasconcellea cauliflora, $V$. parviflora, $\quad V$. cundinamarcensis, $\quad V$. candicans, $V$. quercifoliaand $V$. stipulata (Badillo, 2000).

The symptoms of PRSV can be observed as chlorosis, mosaic, water soaked oily streaks on the petiole and the upper part of the trunk. Shoe string like symptom which sometime due to distortion of young leaves which look like the mite damage symptoms similar to stunting of infected plants and flower abortion. This will cause decreased production as well as quality of the fruit as decrease in sugar level by 50 per cent or more (Purcifull et al., 1984; Gonsalves, 1994).

For the control of the disease caused by PRSV includes control measures such as rouging or removal of infected plants, for some extent cultural practices, cross protection and planting of tolerant cultivars (Gonsalves, 1994). Though these methods are found to be not successful in controlling the disease and its effects. Hence remaining and best method is the development of virus tolerant/resistant cultivars through conventional breeding which can be consistent tool for long-term disease control. In this perspective, very little work has been attempted using genus Vasconcellea, which has the desirable gene for PRSV resistance.

Work on intergeneric hybridization for PRSV tolerance or resistance was carried out by several workers, viz., Muthulakshmi et al., (2007), Chan (2004), Magdalita et al., (1997), Manshardt et al., (1995), Zee (1985), Conover and Litz (1981), however complete success has not been achieved so far and still a variety resistant to PRSV for commercial cultivation is lacking. Hence, work on intergeneric hybridization using commercial cultivars viz., Arka Surya, Arka Prabhath and Red Lady with wild species viz., Vasconcellea cauliflora, $V$. cundinamarcensis and $V$. 
parviflora (after overcoming the crossability barriers using 5 per cent sucrose, pollen germinating media) could help in the generation of progenies tolerant/ resistant to PRSV. Keeping this information as a baseline, an objective was set to know the cross compatibility between two distant genera viz., Carica and Vasconcellea for PRSV tolerance or resistance.

\section{Materials and Methods}

The experiment was carried out at the ICARIndian Institute of Horticultural Research (ICAR-IIHR), Bengaluru located at $13^{\circ} 58^{\prime}$ North latitude and $78^{\circ}$ East longitudes and at an altitude of $890 \mathrm{~m}$ above mean sea level. The soil is red sandy loam with a $\mathrm{pH}$ of 5.26.4. The maximum mean temperature ranges from $27.00^{\circ} \mathrm{C}$ to $35.00^{\circ} \mathrm{C}$ with a mean of $31.00^{\circ} \mathrm{C}$ while, the minimum mean temperature ranges from $10.00^{\circ} \mathrm{C}$ to $21.00^{\circ} \mathrm{C}$ with a mean of $15.50^{\circ} \mathrm{C}$. The mean relative humidity, mean wind speed and total rainfall were 65.00 per cent, $4.38 \mathrm{~km}$ per $\mathrm{hr}$ and $478.70 \mathrm{~mm}$ resp.

This experiment was carried out using three gynodioecious cultivars like Arka Surya, Arka Prabhath and Red Lady as female parents and wild species like Vasconcellea cauliflora, $V$. cundinamarcensis and $V$. parviflora as male parents. The brief descriptions of the parents used in the study are as follows (Plate 1-6).

\section{Arka Prabhath}

An advanced generation hybrid derivative from the cross of (Arka Surya x Tainung-1) $x$ Local Dwarf released from ICAR- Indian Institute of Horticultural Research. It is gynodioecious in nature, with large sized fruits of 900-1200 $\mathrm{g}$ and with smooth skin. The pulp is deep orange red in colour with good keeping quality and high TSS $\left(12-13^{\circ} \mathrm{B}\right)$ (Plate 1).

\section{Arka Surya}

An advanced generation hybrid derivative from the cross of Sunrise Solo x Pink Flesh Sweet released from ICAR-Indian Institute of Horticultural Research. It is gynodioecious in nature, with medium sized fruits weighing about 600-800 g. The pulp is deep pink colour, free from typical papaya odour with high TSS $\left(12-13^{\circ} \mathrm{B}\right)$ and small fruit cavity (Plate 2).

\section{Red lady}

It is a gynodioecious $F_{1}$ hybrid. The plants are semi-dwarf, good yielding, fruits are medium to big size, pulp deep orange-red in colour and sweet in taste (Plate 3).

\section{Vasconcellea cauliflora}

It is a wild species native to Latin America and dioecious in nature. Plants are semidwarf, bearing small fruits weighing 34-35 g, oblong-shaped, ridged and on ripening attain yellow colour but are non-edible. Seeds are having prominent spiny hairy structures with light brown colour (Jimenez and Horovitz, 1958). Plants are reported (Badillo, 2000) to be resistant to virus disease (Plate 4).

\section{Vasconcellea cundinamarcensis}

The mountain papaya is native to Andean regions from Venezuela to Chile at altitudes between 6,000 and 10,000 ft (1,800-3,000 m). It occurs as both monoecious and dioecious forms (Badillo, 2000). It grows on mountains in Ceylon and South India. The plant is stout and tall but bears a small, yellow, five angled fruit of sweet flavour. The fruit is edible but highly rich in papain for eating raw. It is cooked as vegetable and is canned for domestic consumption and also for export. It is reported to be resistant to virus disease and frost (Jimenez and Horovitz, 1958) (Plate 5). 


\section{Vasconcellea parviflora}

This is native to Tropical South America and grown widely in South America. It is an evergreen shrub, dioecious small trees growing up to 50-200 $\mathrm{cm}$ tall. It can grow up to sea level to over 2, $000 \mathrm{~m}$. It bears edible fruit which is harvested from the wild for local use. Fruits are having fragrance and flavour of mombin (Spondias sp.). Fruits are orange in colour, $30-40 \mathrm{~mm}$ long and 10-15 mm wide (Jimenez and Horovitz, 1958) (Plate 6).

Intergeneric hybridization was carried out using Carica papaya varieties viz., Arka Prabhath, Arka Surya and Red Lady as female parents with Vasconcellea species viz., $V$. cauliflora, $V$. cundinamarcensis and $V$. parvifloraas the male parents. In the selected female parents (Plate 7), fully developed and unopened (about to open) female flowers (Plate 8a) were selected and bagged using butter paper bags (Plate $8 b$ ). Pollen grains were collected from the already identified male parents, where the fully developed male flowers were selected and bagged using butter paper bags, in order to avoid contamination by foreign pollen. The pollen grains were collected in the petri plates and were made into a slurry using the nutrient solution (Sucrose 5\% + Boric acid $100 \mathrm{ppm}+\mathrm{MgSO}_{4}$ $200 \mathrm{ppm}+\mathrm{CaNO}_{3} 300 \mathrm{ppm}+\mathrm{KNO}_{3} 100$ ppm) (Plate 8c). Selected female flower was forced to open (Plate 8d) for crossing (Plate $8 \mathrm{e})$.

Pollination was effected by smearing the prepared pollen slurry on to the stigmatic surface (Plate 8f) of the bagged female flowers and were labelled (Plate 8g) and rebagged again (Plate 8h\&i). In order to see the effect of nutrient solution in fruit set, crosses were also made using only pollen grains and without nutrient solution (Plate 8). The details of different cross combinations are given in Table 1.

\section{Statistical analysis}

The data on seed characters were subjected to Fisher's method of analysis of variance (ANOVA) as given by Sundarraj et al., (1972). A simple randomised complete block design was followed with eighteen treatments and two replications. Wherever the $\mathrm{F}$ test was significant for comparison of treatment means, $C D$ values were worked out at 0.05 probability level. The treatment means were separated by using Duncans Multiple Range Test (DMRT). Data obtained were subjected to arc sin transformation.

\section{Results and Discussion}

\section{Flowers crossed (number) and fruit set (\%)}

Across different intergeneric cross combinations, a total of 1802 crosses were made, which included both nutrient solution (928) and control (874). The number of flowers crossed under each cross combination ranged from 89 to 120 depending upon the availability of flowers at the time of crossing.

The data on fruit set recorded among various intergeneric crosses (Table 2) revealed significant differences among the treatments. Out of 1802 crosses made, fruit set ranged from 37.50 to 97.92 per cent. Highest fruit set was recorded in Arka Prabhath x V. cauliflora $\left(\mathrm{T}_{1}, 97.92 \%\right)$ (Control) and and Arka Surya $\mathrm{x}$ $V$. cundinamarcensis $\left(\mathrm{T}_{10}, 97.92 \%\right)$ using nutrient solution which was on par with Arka Surya $\mathrm{x} V$. cundinamarcensis $\left(\mathrm{T}_{10}, 97.92 \%\right)$, followed by Arka Surya x V. cauliflora $\left(\mathrm{T}_{7}\right.$, $96.88 \%)$. The lowest fruit set $\left(\mathrm{T}_{17}, 37.50 \%\right)$ was recorded in Red Lady x $V$. parviflora (Plate 9).

Fruit set is an indication of initial compatibility in intergeneric hybridization. Nutrient solution not only increases seed set but also has some beneficial influence on fruit 
set and fruit growth which can be termed as metaxenia (effect of pollen parent on fruit growth and development). This phenomenon was reported by Abbas and Ibrahim, 1998, in date palm, where pollen parent influences physiology of fruit growth and ripening.

In the present investigation, wide range of fruit set per cent (37.50 to 97.92) was observed among different cross combination. This might be due to differential compatibility of pollen parents with female parents. Among different female and male parents used in the study, Arka Prabhath with V. cauliflora and Arka Surya with $V$. cundinamarcensis were found to be a good combiner which resulted in highest fruit set percentage (97.92). Similar results were obtained by earlier workers using wild species of $V$. cauliflora and $V$. cundinamarcensis as male parents (Dinesh et al., 2007; Bharathi, 2011; Jayavalli et al., 2011 and Azad et al., 2012).

\section{Fruit retention $(\%)$}

The data on fruit retention (Table 2) across various intergeneric crosses exhibited significant difference among the treatments. It ranged from 5.21 to 98.96 per cent among various crosses. Highest fruit retention was recorded in Arka Prabhath x V. cauliflora $\left(\mathrm{T}_{2}\right.$, $98.96 \%$ ) using nutrient solution, which was significantly superior to other treatments. The next best significant treatments were Arka Prabhath $\mathrm{x} V$. cundinamarcensis $\left(\mathrm{T}_{4}, 83.33\right.$ $\%)$ and Arka Prabhath x V. parviflora $\left(\mathrm{T}_{6}\right.$, $73.96 \%$ ) using nutrient solution. The lowest fruit retention $(5.21 \%)$, was recorded in the combination of Arka Surya $\mathrm{x} V$. parviflora $\left(\mathrm{T}_{11}\right)$, Arka Surya $\mathrm{x} V$. cauliflora $\left(\mathrm{T}_{7}, 6.25 \%\right)$ and Red Lady x V. parviflora $\left(\mathrm{T}_{17}, 7.29 \%\right)$ without nutrient solution.

In the present investigations, even though initial fruit set percentage was high (97.92\% in Arka Prabhath X V. cauliflora) in control treatments, the fruit retention was high (98.96 $\%$ in Arka Prabhath X V. cauliflora) when nutrient solution was used for crossing. This could be due to beneficial effect of use of nutrient solution which has helped in further fruit development till maturity. As parthenocarpic fruit development has been noticed in papaya, the trigger of pollen could have helped in initial fruit set and not up to final fruit retention and seed development. This result was in confirmation with Jayavalli et al., 2011, who also recorded higher fruit set in nutrient solution treatments than control.

In case of control treatments, sequential fruit drop was noticed which might be due to improper pollen germination and pollen tube growth. Similar result was obtained by earlier worker (Jayavalli et al., 2011). Among different cross combinations, Arka Prabhath was found to be a good combiner for all male parents which resulted in good fruit set (85.42, 78.13 and $84.38 \%$ in three wild species of nutrient solution treatments) and fruit retention percentage $(98.96,83.33$ and $73.96 \%$ in all three wild species of nutrient solution treatments) as compared to other female parents (Arka Surya and Red Lady). This could be due to genotypic nature of Arka Prabhath which responded very well in intergeneric crosses using wild relatives. As regards male parents both the wild species $V$. cauliflora and $V$. cundinamarcensis helped in high fruit retention compared to wild species V. parviflora (Jimenez and Horovitz, 1958). Similar results were obtained by Jayavalli et al., 2011, where $V$. cauliflora has resulted in good set and final recovery.

\section{Mean number of fruits harvested with fertile seeds}

The data on mean number of fruits harvested with fertile seeds among various intergeneric crosses (Table 3) revealed the significant difference among the treatments, which 
ranged from 0.00 to 30.00 . The significantly highest mean number (30.00) was recorded in the cross Arka Prabhath x V. cauliflora $\left(\mathrm{T}_{2}\right)$ using nutrient solution. The next best treatments were Arka Prabhath $\mathrm{x} V$. cundinamarcensis $\left(\mathrm{T}_{4}, 19.50\right)$ and Arka Prabhath $\mathrm{x} V$. parviflora $\left(\mathrm{T}_{6}, 14.00\right)$. In cross combinations Arka Surya x V. cauliflora $\left(\mathrm{T}_{7}\right)$, Arka Surya x $V$. cundinamarcensis $\left(\mathrm{T}_{9}\right)$, Arka Surya $\mathrm{x} V$. parviflora $\left(\mathrm{T}_{11}\right)$, Red Lady $\mathrm{x} V$. cauliflora $\left(\mathrm{T}_{13}\right)$ and Red Lady $\mathrm{x} V$. parviflora $\left(\mathrm{T}_{17}\right)$ no fruits were produced with fertile seeds.

In the present investigations, highest number of fruits $(30.00,19.50$ and 14.00 in nutrient solution with wild species) with fertile seeds were recovered when Arka Prabhath was used as a female parent irrespective of male parents. It might be due to the better compatibility of Arka Prabhath with Vasconcellea genus and also due to the beneficial influence of nutrient solution in pollen germination and pollen tube growth as demonstrated earlier by Dinesh et al., 2007; Jayavalli et al., 2011 and Lichamo, 2015.

\section{Mean number of seeds per fruit}

The data on mean number of seeds per fruit among various intergeneric crosses (Table 3) revealed significant difference among the treatments, which ranged from 0.00 to 204.14. The significantly highest mean number (204.14) was recorded in the cross Arka Surya $\mathrm{x} V$. cauliflora $\left(\mathrm{T}_{8}\right)$. The next best treatments were Arka Prabhath x $V$. cauliflora $\left(\mathrm{T}_{2}, 165.78\right)$ and Arka Prabhath $\mathrm{x} V$. cundinamarcensis $\left(\mathrm{T}_{4}, 133.89\right)$. In cross combinations Arka Surya $\mathrm{x} V$. cundinamarcensis $\left(\mathrm{T}_{9}\right)$, Arka Surya $\mathrm{x} V$. parviflora $\left(\mathrm{T}_{11}\right)$ and Red Lady x $V$. parviflora $\left(\mathrm{T}_{17}\right)$ no seeds were produced (Plate 10-12). In the present investigations, highest number of seeds was recovered (204.14) in Arka Surya $\mathrm{x} V$. cauliflora followed by (165.78)
Arka Prabhath X V. cauliflora when nutrient solution was used than control. The reason might be due to component of nutrient solution viz., sucrose (major source of carbohydrate) and micronutrients. Both sucrose and micronutrients are reported to improve pollen germination and pollen tube growth ultimately resulting good seed set in intergeneric crossing (Dinesh et al., 2007). This indicates efficiency of nutrient solution in complete development of embryo upon intergeneric hybridization. In the earlier study by Jayavalli, (2011) the combination resulted in high (400) number of seeds per fruit CP 50 $\mathrm{X}$ V. cauliflora.

\section{Mean number of fertile seeds per fruit}

The data on mean number of fertile seeds per fruit recorded among various intergeneric crosses presented (Table 3) revealed significant differences, which was in the range of 0.00 to 70.82 . The significantly highest mean number (70.82) of fertile seeds per fruit were recovered in the combination Arka Prabhath $\mathrm{x} V$. cundinamarcensis $\left(\mathrm{T}_{4}\right)$ followed by Arka Prabhath x $V$. cauliflora $\left(\mathrm{T}_{2}, 63.99\right)$. The next best treatments were and Arka Surya $\mathrm{x} V$. cundinamarcensis $\left(\mathrm{T}_{10}\right.$, 28.50) and Arka Prabhath x V. parviflora $\left(\mathrm{T}_{6}\right.$, 28.14) using nutrient solution. No seeds were recovered in the combinations viz., Arka Surya $\mathrm{x} V$. cauliflora $\left(\mathrm{T}_{7}\right)$, Arka Surya $\mathrm{x} V$. cundinamarcensis $\left(\mathrm{T}_{9}\right)$, Arka Surya $\mathrm{x} V$. parviflora $\left(\mathrm{T}_{11}\right)$, Red Lady $\mathrm{x} V$. cauliflora $\left(\mathrm{T}_{13}\right)$ and Red Lady x V. parviflora $\left(\mathrm{T}_{15}\right)$.

In the present study, it was observed that the use of nutrient solution proved its superiority in producing good number of fertile seeds (70.82 and 63.99 in Arka Prabhath crosses). This might be due to micronutrients results in chemotropism of pollens towards ovaries in presence of glucose and water ultimately helps in better pollen germination (Reger et al., 1992). $\mathrm{F}_{1}$ seeds of most of cross 
combinations without nutrient solution resulted in infertile seeds (non-viable) (Mekako and Nakasone, 1975). This may be due to post zygotic embryo abortion due to improper pollen tube growth and lack of food reserve (endosperm) for embryo development which occurred in control treatments (Manshardt and Wenslaff, 1989). However sufficient carbohydrate source (sucrose) and micronutrient had helped in getting high number of fertile seeds in flowers crossed with help of nutrient solution.

Germination percentage of $F_{1}$ intergeneric progenies

The data on germination percentage of $F_{0}$ intergeneric progenies among various intergeneric crosses (Table 4) revealed significant differences, which was in the range of 0.00 to 82.29 .

The significantly highest germination $(82.29$ $\%)$ was recorded in the cross Red Lady x $V$. cundinamarcensis which was on par with Red Lady $x V$. parviflora $\left(\mathrm{T}_{18}, 50.00 \%\right)$. The next best treatment was Red Lady x $V$. cauliflora $\left(\mathrm{T}_{14}, 30.00 \%\right)$. As no seeds were recovered in the treatment combinations viz., Arka Surya $\mathrm{x}$ $V$. cauliflora $\left(\mathrm{T}_{7}\right)$, Arka Surya $\mathrm{x} V$. cundinamarcensis $\left(\mathrm{T}_{9}\right)$, Arka Surya $\mathrm{x} V$. parviflora $\left(\mathrm{T}_{11}\right)$, Red Lady $\mathrm{x}$ V. cauliflora $\left(\mathrm{T}_{13}\right)$ and Red Lady x V. parviflora $\left(\mathrm{T}_{15}\right)$, no seedlings were obtained.

Table.1 Details of cross combinations

\begin{tabular}{|c|l|}
\hline Treatments & \multicolumn{1}{|c|}{ Cross combination } \\
\hline $\mathbf{T}_{\mathbf{1}}$ & Arka Prabhath $\times$ Vasconcellea cauliflora \\
\hline $\mathbf{T}_{\mathbf{2}}$ & Arka Prabhath $\times$ Vasconcellea cauliflora \\
\hline $\mathbf{T}_{\mathbf{3}}$ & Arka Prabhath $\times$ Vasconcellea cundinamarcensis \\
\hline $\mathbf{T}_{\mathbf{4}}$ & Arka Prabhath $\times$ Vasconcellea cundinamarcensis \\
\hline $\mathbf{T}_{\mathbf{5}}$ & Arka Prabhath $\times$ Vasconcellea parviflora \\
\hline $\mathbf{T}_{\mathbf{6}}$ & Arka Prabhath $\times$ Vasconcellea parviflora \\
\hline $\mathbf{T}_{\mathbf{7}}$ & Arka Surya $\times$ Vasconcellea cauliflora \\
\hline $\mathbf{T}_{\mathbf{8}}$ & Arka Surya $\times$ Vasconcellea cauliflora \\
\hline $\mathbf{T}_{\mathbf{9}}$ & Arka Surya $\times$ Vasconcellea cundinamarcensis \\
\hline $\mathbf{T}_{\mathbf{1 0}}$ & Arka Surya $\times$ Vasconcellea cundinamarcensis \\
\hline $\mathbf{T}_{\mathbf{1 1}}$ & Arka Surya $\times$ Vasconcellea parviflora \\
\hline $\mathbf{T}_{\mathbf{1 2}}$ & Arka Surya $\times$ Vasconcellea parviflora \\
\hline $\mathbf{T}_{\mathbf{1 3}}$ & Red Lady $\times$ Vasconcellea cauliflora \\
\hline $\mathbf{T}_{\mathbf{1 4}}$ & Red Lady $\times$ Vasconcellea cauliflora \\
\hline $\mathbf{T}_{\mathbf{1 5}}$ & Red Lady $\times$ Vasconcellea cundinamarcensis \\
\hline $\mathbf{T}_{\mathbf{1 6}}$ & Red Lady $\times$ Vasconcellea cundinamarcensis \\
\hline $\mathbf{T}_{\mathbf{1 7}}$ & Red Lady $\times$ Vasconcellea parviflora \\
\hline $\mathbf{T}_{\mathbf{1 8}}$ & Red Lady $\times$ Vasconcellea parviflora \\
\hline Replication & Two \\
\hline Number of flowers per & 50 \\
\hline replication & \\
\hline Design & Randomised complete block design $($ RCBD) \\
\hline
\end{tabular}


Table.2 Details on number of flowers crossed, fruit set (\%) and fruit retention (\%) in various intergeneric crosses

\begin{tabular}{|c|c|c|c|}
\hline Treatment & $\begin{array}{c}\text { Number of } \\
\text { flowers crossed }\end{array}$ & $\begin{array}{l}\text { Fruit set } \\
\quad(\%)\end{array}$ & $\begin{array}{c}\text { Fruit } \\
\text { retention }(\%)\end{array}$ \\
\hline$T_{1}$ : Arka Prabhath $x$ V. cauliflora (Control) & 95 & $\begin{array}{c}97.92 \\
(83.90)^{\mathrm{a}}\end{array}$ & $\begin{array}{c}28.13 \\
(32.02)^{\text {ef }}\end{array}$ \\
\hline $\begin{array}{l}\mathrm{T}_{2}: \begin{array}{l}\text { Arka Prabhath } \mathrm{x} \text { V. cauliflora (Nutrient } \\
\text { solution) }\end{array}\end{array}$ & 120 & $\begin{array}{c}85.42 \\
(67.59)^{\text {bcde }}\end{array}$ & $\begin{array}{c}98.96 \\
(85.64)^{\mathrm{a}}\end{array}$ \\
\hline $\begin{array}{l}\mathbf{T}_{3}: \text { Arka Prabhath } \mathrm{x} V \text {. cundinamarcensis } \\
\text { (Control) }\end{array}$ & 93 & $\begin{array}{c}67.71 \\
(55.44)^{\text {efgh }}\end{array}$ & $\begin{array}{c}42.71 \\
(40.80)^{d}\end{array}$ \\
\hline $\begin{array}{l}\mathrm{T}_{4}: \text { Arka Prabhath } \mathrm{x} V \text {. cundinamarcensis } \\
\text { (Nutrient solution) }\end{array}$ & 125 & $\begin{array}{c}78.13 \\
(62.27)^{\operatorname{defg}}\end{array}$ & $\begin{array}{c}83.33 \\
(65.90)^{\mathrm{b}}\end{array}$ \\
\hline $\mathrm{T}_{5}:$ Arka Prabhath x V.parviflora (Control) & 94 & $\begin{array}{c}59.38 \\
(50.41)^{\mathrm{ghi}}\end{array}$ & $\begin{array}{c}32.29 \\
(34.62)^{\mathrm{e}}\end{array}$ \\
\hline $\begin{array}{l}\mathrm{T}_{6}: \text { Arka Prabhath } \mathrm{x} V \text {. parviflora (Nutrient } \\
\text { solution) }\end{array}$ & 120 & $\begin{array}{c}84.38 \\
(66.81)^{\text {cdef }}\end{array}$ & $\begin{array}{c}73.96 \\
(59.32)^{\mathrm{c}}\end{array}$ \\
\hline $\mathbf{T}_{7}$ : Arka Surya x V. cauliflora (Control) & 98 & $\begin{array}{c}96.88 \\
(82.55)^{\mathrm{ab}}\end{array}$ & $\begin{array}{c}6.25 \\
(14.47)^{\mathrm{j}}\end{array}$ \\
\hline $\begin{array}{l}T_{8}: \text { Arka Surya } x \text { V. cauliflora (Nutrient } \\
\text { solution) }\end{array}$ & 96 & $\begin{array}{c}85.42 \\
(67.59)^{\text {bdde }}\end{array}$ & $\begin{array}{c}19.79 \\
(26.40)^{\mathrm{gh}}\end{array}$ \\
\hline $\begin{array}{l}\text { T9: Arka Surya x V. cundinamarcensis } \\
\text { (Control) }\end{array}$ & 102 & $\begin{array}{c}80.21 \\
(66.97)^{\text {cdef }}\end{array}$ & $\begin{array}{c}8.33 \\
(16.65) \mathrm{j}\end{array}$ \\
\hline $\begin{array}{l}T_{10}: \text { Arka Surya } x V . \text { cundinamarcensis } \\
\text { (Nutrient solution) }\end{array}$ & 97 & $\begin{array}{c}97.92 \\
(83.90)^{\mathrm{a}}\end{array}$ & $\begin{array}{c}14.58 \\
(22.40)^{\mathrm{hi}}\end{array}$ \\
\hline$T_{11}$ : Arka Surya x $V$. parviflora (Control) & 92 & $\begin{array}{c}61.46 \\
(51.75)^{\text {fghi }}\end{array}$ & $\begin{array}{c}5.21 \\
(13.13)^{\mathrm{j}}\end{array}$ \\
\hline $\begin{array}{l}T_{12}: \text { Arka Surya } x V \cdot \text { parviflora (Nutrient } \\
\text { solution) }\end{array}$ & 94 & $\begin{array}{c}95.83 \\
(78.61)^{\mathrm{abc}}\end{array}$ & $\begin{array}{c}8.33 \\
(16.77)^{\mathrm{j}}\end{array}$ \\
\hline$T_{13}:$ Red Lady x $V$. cauliflora (Control) & 100 & $\begin{array}{c}88.54 \\
(70.71)^{\text {abcd }}\end{array}$ & $\begin{array}{c}13.54 \\
(21.46)^{\mathrm{i}}\end{array}$ \\
\hline $\begin{array}{l}T_{14}: \text { Red Lady } x V \text {. cauliflora } \text { (Nutrient } \\
\text { solution) }\end{array}$ & 89 & $\begin{array}{c}76.04 \\
(60.95)^{\operatorname{defg}}\end{array}$ & $\begin{array}{c}43.75 \\
(41.40)^{d}\end{array}$ \\
\hline $\begin{array}{l}T_{15}: \text { Red Lady } x \text { V. cundinamarcensis } \\
\text { (Control) }\end{array}$ & 102 & $\begin{array}{c}60.42 \\
(51.01)^{\mathrm{ghi}}\end{array}$ & $\begin{array}{c}22.92 \\
(28.60)^{\mathrm{fg}}\end{array}$ \\
\hline $\begin{array}{l}T_{16}: \text { Red Lady } x V \text {. cundinamarcensis } \\
\text { (Nutrient solution) }\end{array}$ & 104 & $\begin{array}{c}42.71 \\
(40.79)^{\text {hi }}\end{array}$ & $\begin{array}{c}43.75 \\
(41.40)^{\mathrm{d}}\end{array}$ \\
\hline$T_{17}:$ Red Lady x $V$. parviflora (Control) & 98 & $\begin{array}{c}37.50 \\
(37.75)^{\mathrm{i}}\end{array}$ & $\begin{array}{c}7.29 \\
(15.62)^{j}\end{array}$ \\
\hline $\begin{array}{l}T_{18}: \begin{array}{l}\text { Red Lady } x ~ \\
\text { solution) }\end{array}\end{array}$ & 83 & $\begin{array}{c}84.38 \\
(66.72)^{\text {cdef }}\end{array}$ & $\begin{array}{c}48.96 \\
(44.40)^{\mathrm{d}}\end{array}$ \\
\hline Total & 1802.00 & - & - \\
\hline CV (\%) & 0.11 & 11.34 & 6.15 \\
\hline $\mathrm{CD}(\mathrm{p}=\mathbf{0 . 0 5})$ & - & 15.23 & 4.48 \\
\hline SEm \pm & - & 5.1 & 1.5 \\
\hline
\end{tabular}

Values in parenthesis indicate arc sin transformed ones

Values following same alphabets in the column are not significantly different at $\mathrm{P}=0.05$ 
Table.3 Details on mean number of fruits harvested with fertile seeds, mean number of seeds per fruit and mean number of fertile seeds per fruit in various intergeneric crosses

\begin{tabular}{|c|c|c|c|}
\hline Treatment & $\begin{array}{l}\text { Mean number } \\
\text { of fruits with } \\
\text { fertile seeds }\end{array}$ & $\begin{array}{l}\text { Mean number } \\
\text { of seeds per } \\
\text { fruit }\end{array}$ & $\begin{array}{c}\text { Mean number } \\
\text { of fertile seeds } \\
\text { per fruit }\end{array}$ \\
\hline $\mathrm{T}_{1}$ : Arka Prabhath x V. cauliflora (Control) & $\begin{array}{c}5.00 \\
(2.33)^{\mathrm{d}}\end{array}$ & $\begin{array}{l}56.76 \\
(7.56)^{d}\end{array}$ & $\begin{array}{c}20.91 \\
(4.77)^{\mathrm{bc}}\end{array}$ \\
\hline $\mathrm{T}_{2}$ : Arka Prabhath $\mathrm{x}$ V. cauliflora (Nutrient solution) & $\begin{array}{c}30.00 \\
(5.51)^{\mathrm{a}}\end{array}$ & $\begin{array}{c}165.78 \\
(12.89)^{b}\end{array}$ & $\begin{array}{c}63.99 \\
(8.02)^{\mathrm{a}}\end{array}$ \\
\hline $\mathbf{T}_{3}$ : Arka Prabhath x V.cundinamarcensis (Control) & $\begin{array}{c}1.00 \\
(1.22)^{\mathrm{ghi}}\end{array}$ & $\begin{array}{c}4.40 \\
(2.21)^{\mathrm{ij}}\end{array}$ & $\begin{array}{c}5.00 \\
(2.33)^{\text {ef }}\end{array}$ \\
\hline $\mathrm{T}_{4}$ : Arka Prabhath $\mathrm{x} V$. cundinamarcensis (Nutrient solution) & $\begin{array}{c}19.50 \\
(4.47)^{b}\end{array}$ & $\begin{array}{c}133.89 \\
(11.56)^{\mathrm{bc}}\end{array}$ & $\begin{array}{c}70.82 \\
(8.42)^{\mathrm{a}}\end{array}$ \\
\hline $\mathrm{T}_{5}$ : Arka Prabhath x V. parviflora (Control) & $\begin{array}{c}2.00 \\
(1.58)^{\mathrm{efg}}\end{array}$ & $\begin{array}{c}18.21 \\
(4.32)^{\mathrm{g}}\end{array}$ & $\begin{array}{c}3.50 \\
(1.99)^{\mathrm{fg}}\end{array}$ \\
\hline$T_{6}:$ Arka Prabhath $x$ V.parviflora (Nutrient solution) & $\begin{array}{c}14.00 \\
(3.80)^{\mathrm{c}}\end{array}$ & $\begin{array}{c}47.57 \\
(6.93)^{\mathrm{de}}\end{array}$ & $\begin{array}{c}28.14 \\
(5.35)^{b}\end{array}$ \\
\hline $\mathbf{T}_{7}$ : Arka Surya x V. cauliflora (Control) & $\begin{array}{c}0.00 \\
(0.70)^{\mathrm{j}}\end{array}$ & $\begin{array}{c}37.66 \\
(6.16)^{\mathrm{ef}}\end{array}$ & $\begin{array}{c}0.00 \\
(0.70)^{\mathrm{h}}\end{array}$ \\
\hline$T_{8}$ : Arka Surya x V. cauliflora (Nutrient solution) & $\begin{array}{c}2.50 \\
(0.72)^{\text {ef }}\end{array}$ & $\begin{array}{c}204.14 \\
(14.27)^{\mathrm{a}}\end{array}$ & $\begin{array}{c}11.00 \\
(3.38)^{\mathrm{de}}\end{array}$ \\
\hline$T_{9}$ : Arka Surya x V. cundinamarcensis (Control) & $\begin{array}{c}0.00 \\
(0.70)^{\mathrm{j}}\end{array}$ & $\begin{array}{c}0.00 \\
(0.70)^{\mathrm{k}}\end{array}$ & $\begin{array}{c}0.00 \\
(0.70)^{\mathrm{h}}\end{array}$ \\
\hline$T_{10}$ : Arka Surya $x$ V. cundinamarcensis (Nutrient solution) & $\begin{array}{c}1.00 \\
(1.22)^{\text {ghi }}\end{array}$ & $\begin{array}{c}120.90 \\
(10.97)^{\mathrm{c}}\end{array}$ & $\begin{array}{c}28.50 \\
(5.37)^{b}\end{array}$ \\
\hline$T_{11}:$ Arka Surya x $V \cdot$ parviflora (Control) & $\begin{array}{c}0.00 \\
(0.70)^{\mathrm{j}}\end{array}$ & $\begin{array}{c}0.00 \\
(0.70)^{\mathrm{k}}\end{array}$ & $\begin{array}{c}0.00 \\
(0.70)^{\mathrm{h}}\end{array}$ \\
\hline$T_{12}:$ Arka Surya x $V \cdot$ parviflora (Nutrient solution) & $\begin{array}{c}1.50 \\
(0.40)^{\text {fgh }}\end{array}$ & $\begin{array}{c}0.50 \\
(1.00)^{\mathrm{jk}}\end{array}$ & $\begin{array}{c}3.00 \\
(1.87)^{\text {fgh }}\end{array}$ \\
\hline$T_{13}:$ Red Lady x $V$. cauliflora (Control) & $\begin{array}{c}0.00 \\
(0.70)^{\mathrm{j}}\end{array}$ & $\begin{array}{c}31.62 \\
(5.98)^{\mathrm{ef}}\end{array}$ & $\begin{array}{c}0.00 \\
(0.70)^{\mathrm{h}}\end{array}$ \\
\hline$T_{14}:$ Red Lady $x$ V. cauliflora (Nutrient solution) & $\begin{array}{c}1.00 \\
(1.14)^{\mathrm{hi}}\end{array}$ & $\begin{array}{c}14.79 \\
(3.89)^{\mathrm{gh}}\end{array}$ & $\begin{array}{c}4.50 \\
(1.89)^{\mathrm{fg}}\end{array}$ \\
\hline$T_{15}:$ Red Lady $x$ V. cundinamarcensis (Control) & $\begin{array}{c}0.50 \\
(0.96)^{\mathrm{ij}}\end{array}$ & $\begin{array}{c}8.09 \\
(2.92)^{\mathrm{hi}}\end{array}$ & $\begin{array}{c}1.00 \\
(1.14)^{\text {gh }}\end{array}$ \\
\hline$T_{16}:$ Red Lady x $V$. cundinamarcensis (Nutrient solution) & $\begin{array}{c}3.00 \\
(1.85)^{\mathrm{e}}\end{array}$ & $\begin{array}{c}24.09 \\
(4.93)^{\mathrm{fg}}\end{array}$ & $\begin{array}{c}13.00 \\
(3.60)^{\mathrm{cd}}\end{array}$ \\
\hline$T_{17}:$ Red Lady $x$ V.parviflora (Control) & $\begin{array}{c}0.00 \\
(0.70)^{\mathrm{j}}\end{array}$ & $\begin{array}{c}0.00 \\
(0.70)^{\mathrm{k}}\end{array}$ & $\begin{array}{c}0.00 \\
(0.70)^{\mathrm{h}}\end{array}$ \\
\hline$T_{18}:$ Red Lady $x$ V.parviflora (Nutrient solution) & $\begin{array}{c}0.50 \\
(0.96)^{\mathrm{ij}}\end{array}$ & $\begin{array}{c}6.72 \\
(2.68)^{\mathrm{hi}}\end{array}$ & $\begin{array}{c}0.50 \\
(0.96)^{\text {gh }}\end{array}$ \\
\hline CV $(\%)$ & 10.60 & 11.51 & 19.17 \\
\hline CD $(p=0.05)$ & 0.39 & 1.35 & 1.18 \\
\hline SEm \pm & 0.12 & 0.45 & 0.39 \\
\hline
\end{tabular}

Values in parenthesis indicate arc sin transformed ones

Values following same alphabets in the column are not significantly different at $\mathrm{P}=0.05$ 
Table.4 Details on germination (\%) of F1 intergeneric hybrid progenies and yield of crossed fruits recorded under various intergeneric crosses

\begin{tabular}{|c|c|c|}
\hline Treatment & $\begin{array}{c}\text { Germination } \\
(\%)\end{array}$ & $\begin{array}{l}\text { Yield of crossed } \\
\text { fruits }(\mathrm{kg} / \text { per tree })\end{array}$ \\
\hline $\mathrm{T}_{1}$ : Arka Prabhath $\mathrm{x}$ V. cauliflora (Control) & $\begin{array}{l}0.00 \\
(0.10)^{\mathrm{c}}\end{array}$ & $13.50^{\mathrm{ef}}$ \\
\hline $\mathrm{T}_{2}$ : Arka Prabhath $\mathrm{x}$ V. cauliflora (Nutrient solution) & $\begin{array}{l}1.02 \\
(5.77)^{\mathrm{c}}\end{array}$ & $48.50^{\mathrm{a}}$ \\
\hline$T_{3}$ : Arka Prabhath $x$ V. cundinamarcensis (Control) & $\begin{array}{l}0.00 \\
(0.10)^{\mathrm{c}}\end{array}$ & $20.50^{\mathrm{d}}$ \\
\hline $\begin{array}{l}\mathrm{T}_{4}: \text { Arka Prabhath } \mathrm{x} V \text {. cundinamarcensis (Nutrient } \\
\text { solution) }\end{array}$ & $\begin{array}{c}3.20 \\
(10.30)^{\mathrm{bc}}\end{array}$ & $40.00^{b}$ \\
\hline $\mathrm{T}_{5}:$ Arka Prabhath $\mathrm{x}$ V. parviflora (Control) & $\begin{array}{l}0.00 \\
(0.10)^{\mathrm{c}}\end{array}$ & $15.50^{\mathrm{e}}$ \\
\hline $\mathrm{T}_{6}$ : Arka Prabhath x V. parviflora (Nutrient solution) & $\begin{array}{c}3.81 \\
(11.24)^{\mathrm{bc}}\end{array}$ & $38.00^{\mathrm{b}}$ \\
\hline $\mathrm{T}_{7}$ : Arka Surya x V. cauliflora (Control) & $\begin{array}{c}0.00 \\
(0.10)^{\mathrm{c}}\end{array}$ & $3.00^{\mathrm{i}}$ \\
\hline $\mathrm{T}_{8}$ : Arka Surya x $V$. cauliflora (Nutrient solution) & $\begin{array}{c}0.00 \\
(0.10)^{\mathrm{c}}\end{array}$ & $9.50^{\text {gh }}$ \\
\hline$T_{9}$ : Arka Surya $x$ V. cundinamarcensis (Control) & $\begin{array}{c}0.00 \\
(0.10)^{\mathrm{c}}\end{array}$ & $4.00^{\mathrm{i}}$ \\
\hline $\begin{array}{l}\mathrm{T}_{10} \text { : Arka Surya } \mathrm{x} V \text {. cundinamarcensis (Nutrient } \\
\text { solution) }\end{array}$ & $\begin{array}{c}0.00 \\
(0.10)^{\mathrm{c}}\end{array}$ & $7.00^{\mathrm{h}}$ \\
\hline$T_{11}$ : Arka Surya x $V$. parviflora (Control) & $\begin{array}{l}0.00 \\
(0.10)^{\mathrm{c}}\end{array}$ & $2.50^{\mathrm{i}}$ \\
\hline$T_{12}:$ Arka Surya $x$ V. parviflora (Nutrient solution) & $\begin{array}{l}0.00 \\
(0.10)^{\mathrm{c}}\end{array}$ & $4.00^{\mathrm{i}}$ \\
\hline$T_{13}:$ Red Lady $x$ V. cauliflora (Control) & $\begin{array}{l}0.00 \\
(0.10)^{\mathrm{c}}\end{array}$ & $8.50^{\text {gh }}$ \\
\hline$T_{14}:$ Red Lady x V. cauliflora (Nutrient solution) & $\begin{array}{c}30.00 \\
(25.43)^{\mathrm{bc}}\end{array}$ & $21.00^{\mathrm{cd}}$ \\
\hline$T_{15}:$ Red Lady x $V$. cundinamarcensis (Control) & $\begin{array}{l}0.00 \\
(0.10)^{\mathrm{c}}\end{array}$ & $11.00^{\mathrm{fg}}$ \\
\hline $\begin{array}{l}T_{16}: \text { Red Lady } x V . \text { cundinamarcensis (Nutrient } \\
\text { solution) }\end{array}$ & $\begin{array}{c}82.29 \\
(71.68)^{\mathrm{a}}\end{array}$ & $21.00^{\mathrm{cd}}$ \\
\hline$T_{17}:$ Red Lady $x$ V. parviflora (Control) & $\begin{array}{c}0.00 \\
(0.10)^{\mathrm{c}}\end{array}$ & $3.50^{\mathrm{i}}$ \\
\hline$T_{18}:$ Red Lady $x$ V. parviflora (Nutrient solution) & $\begin{array}{c}50.00 \\
(45.00)^{\mathrm{ab}}\end{array}$ & $23.50^{\mathrm{c}}$ \\
\hline $\mathrm{CV}(\%)$ & 182.35 & 8.41 \\
\hline $\mathrm{CD}(\mathrm{p}=\mathbf{0 . 0 5})$ & 36.49 & 2.90 \\
\hline S.Em \pm & 12.23 & 0.96 \\
\hline
\end{tabular}

Values in parenthesis indicate arc sin transformed ones

Values following same alphabets in the column are not significantly different at $\mathrm{P}=0.05$ 
Plate.1 Female parent (Arka Prabhath) used in hybridization: a) Fully grown tree, b)Unopened female flower bud, c) Female flower, d) Fruit, e) L.S. of fruit, f) C.S. of fruit

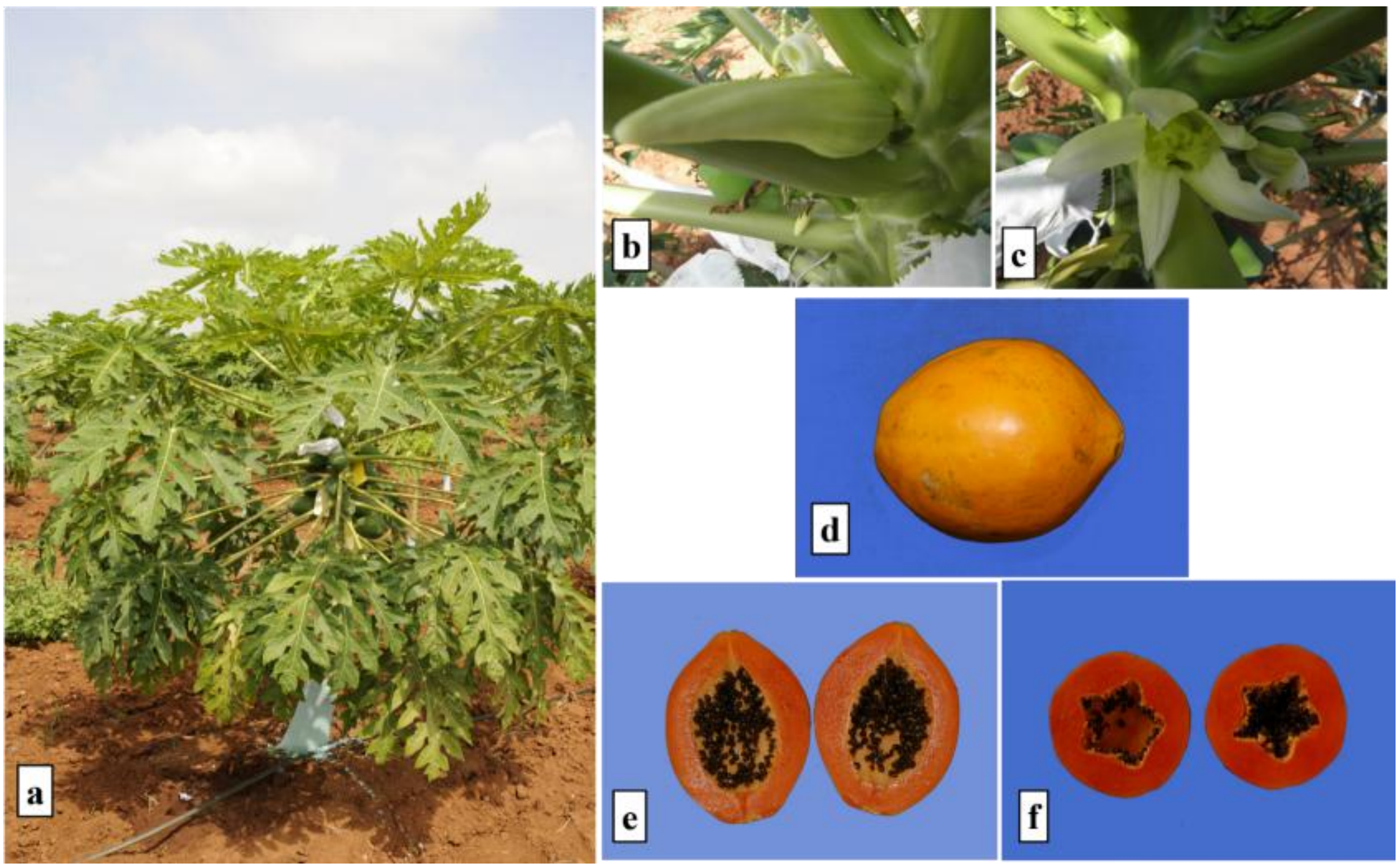


Plate.2 Female parent (Arka Surya) used in hybridization; a) Fully grown tree, b) Unopened female flower bud, c) Female flower, d) Fruit, e) L.S. of fruit, f) C.S. of fruit

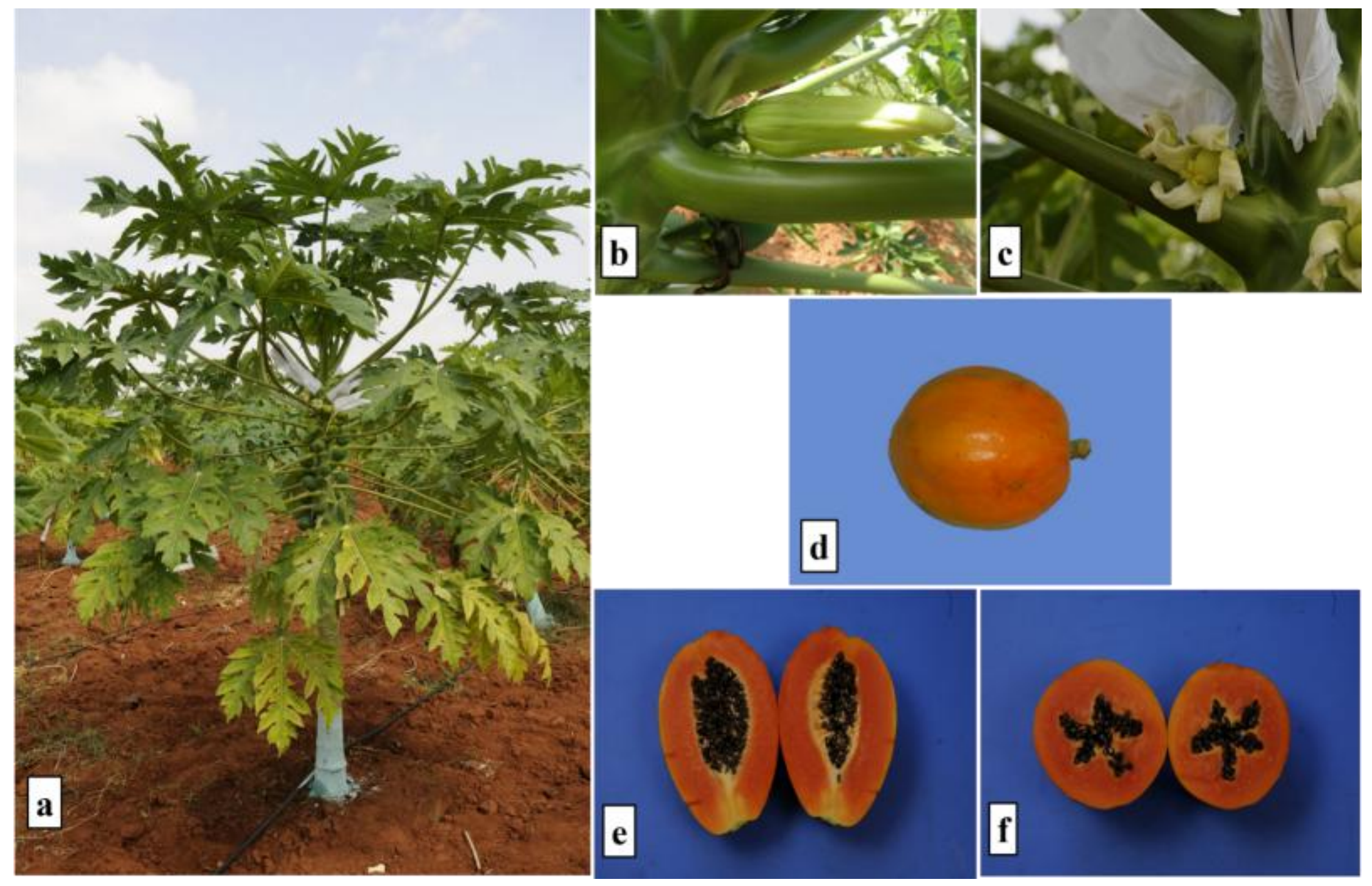


Plate.3 Female parent (Red Lady) used in hybridization: a) Fully grown tree, b) Unopened female flower bud, c) Female flower, d) Fruit, e) L.S. of fruit, f) C.S. of fruit

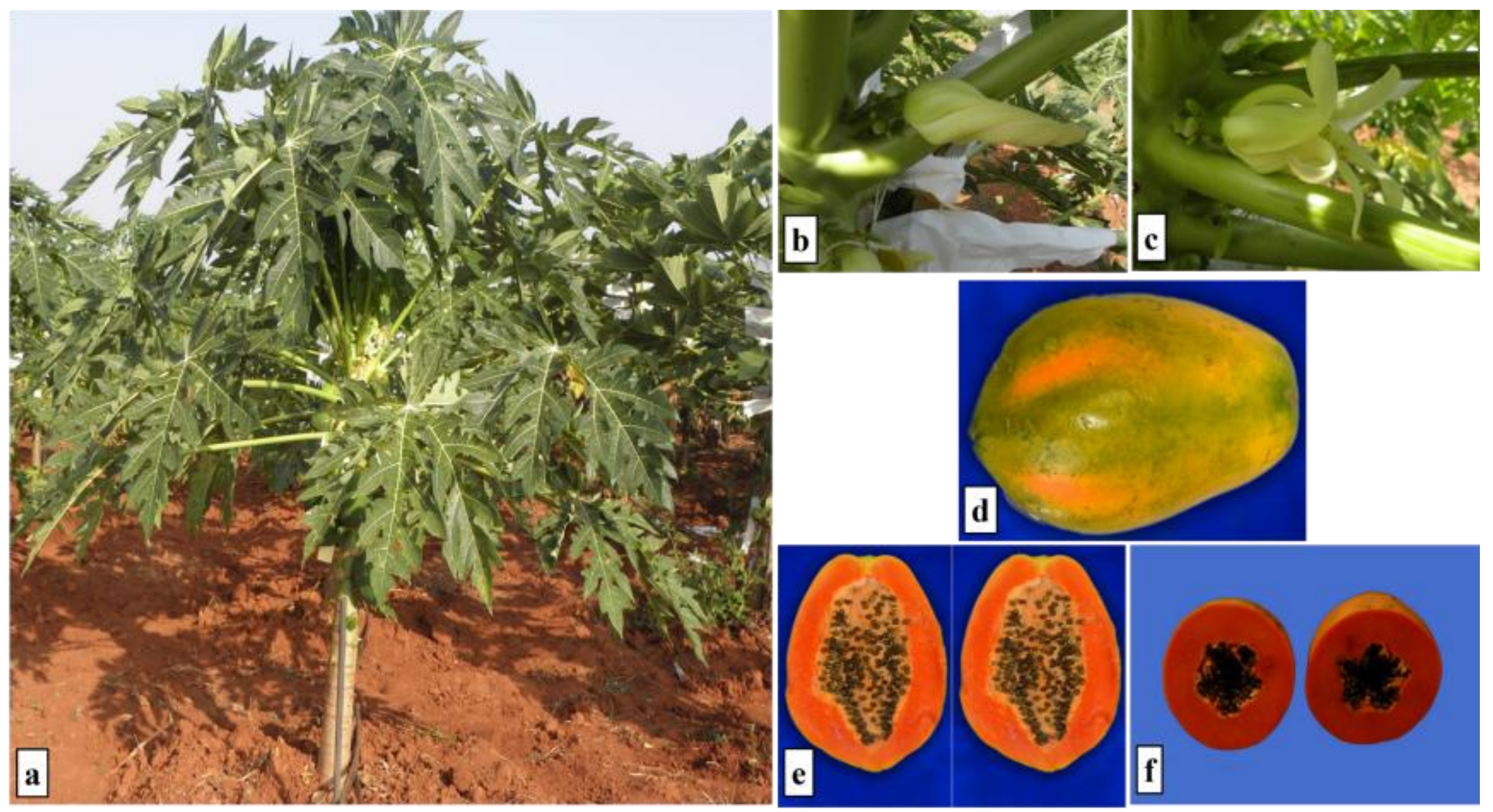


Plate.4 Male parent (Vasconcellea cauliflora) used in hybridization :a) Fully grown male tree, b) Unopened male flower bud, c) Male flower, d) Fruit, e) Fully grown female
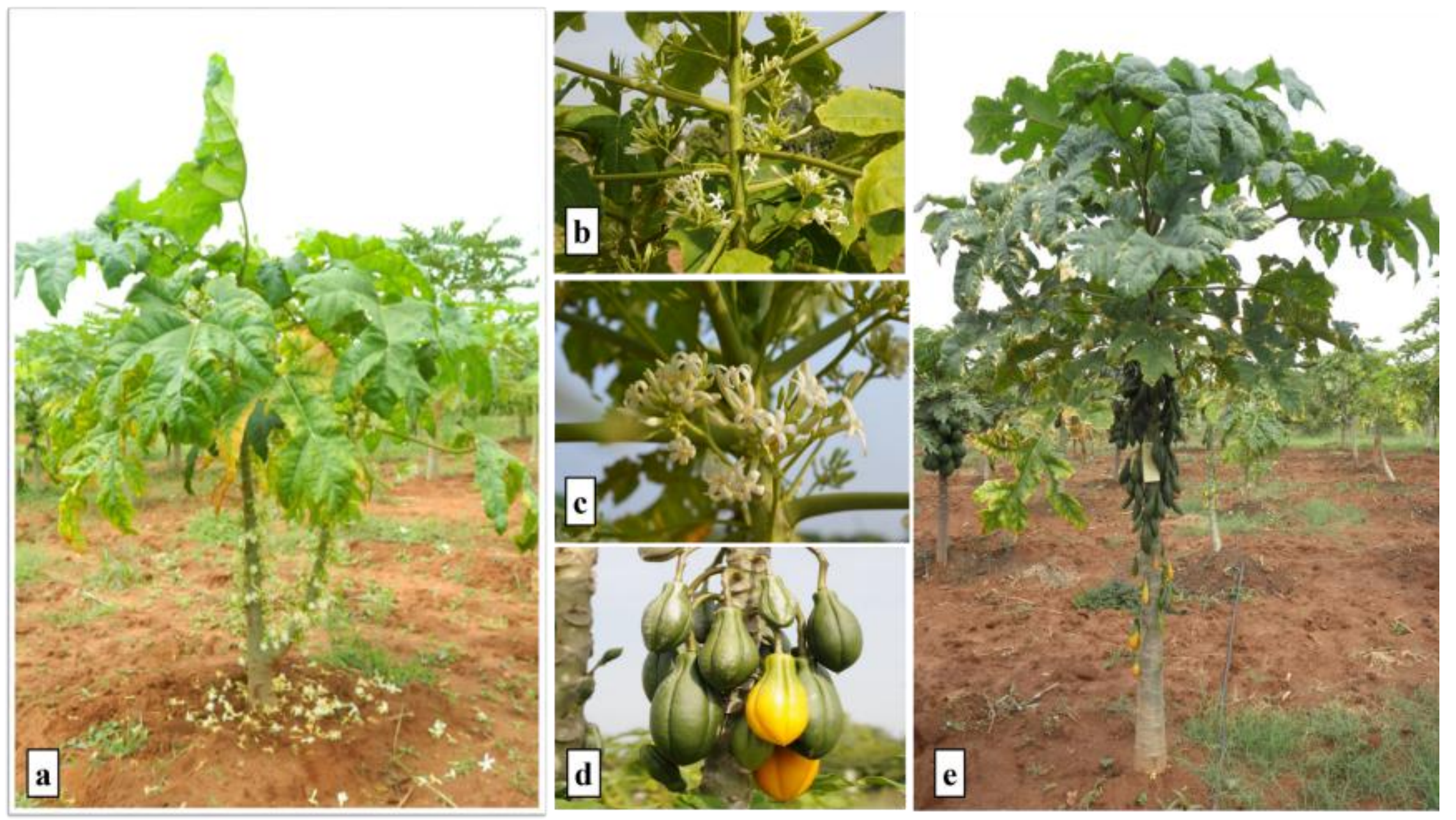
Plate.5 Male parent (Vasconcellea cundinamarcensis) used in hybridization: a) Fully grown male tree, b) Unopened male flower bud, c) Male flower, d) Fruit, e) Fully grown female

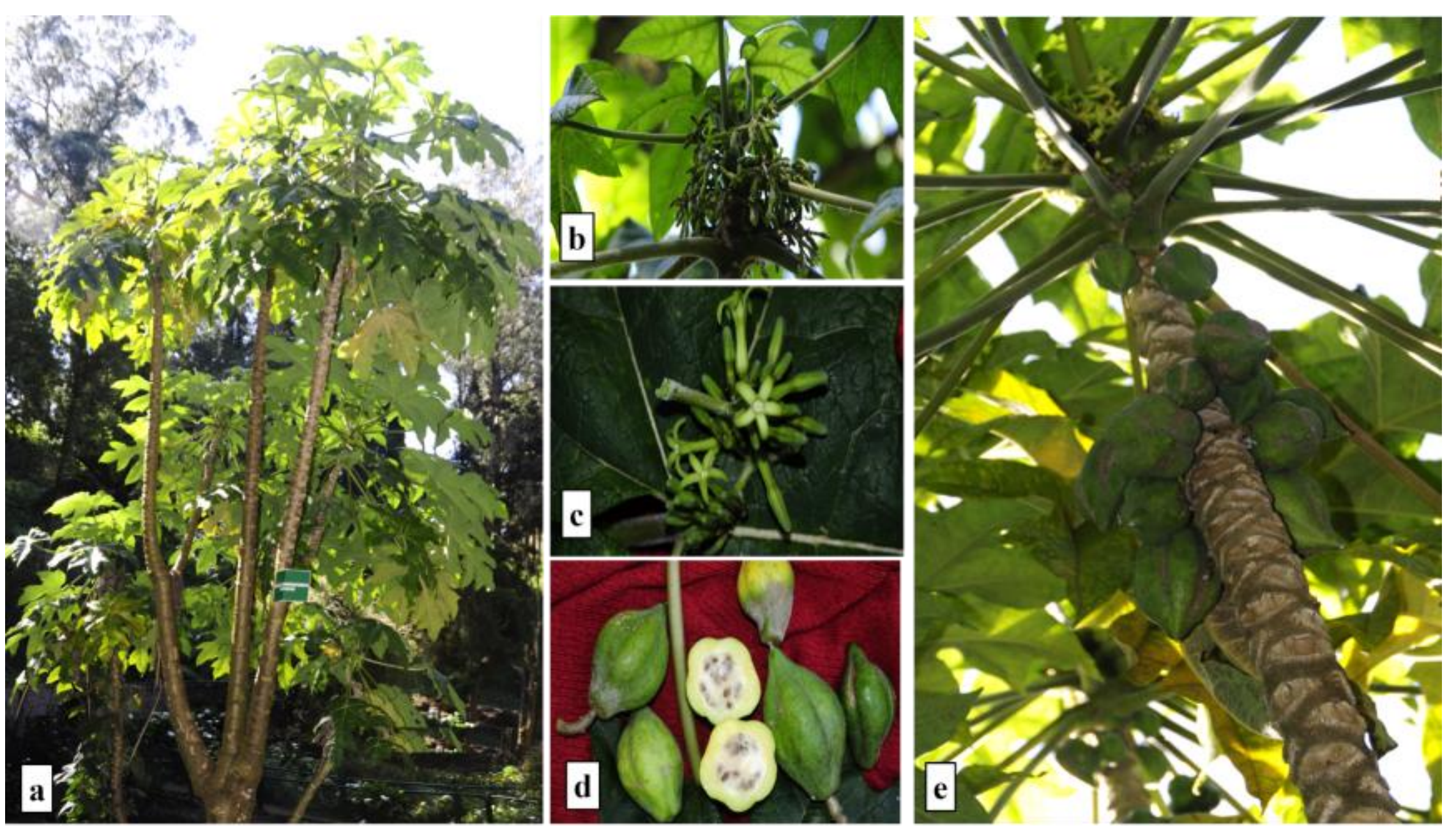


Plate.6 Male parent (Vasconcellea parviflora) used in hybridization: a) Fully grown male tree, b) Unopened male flower bud, c) Male flower, d) Fruit, e) Fully grown female tree

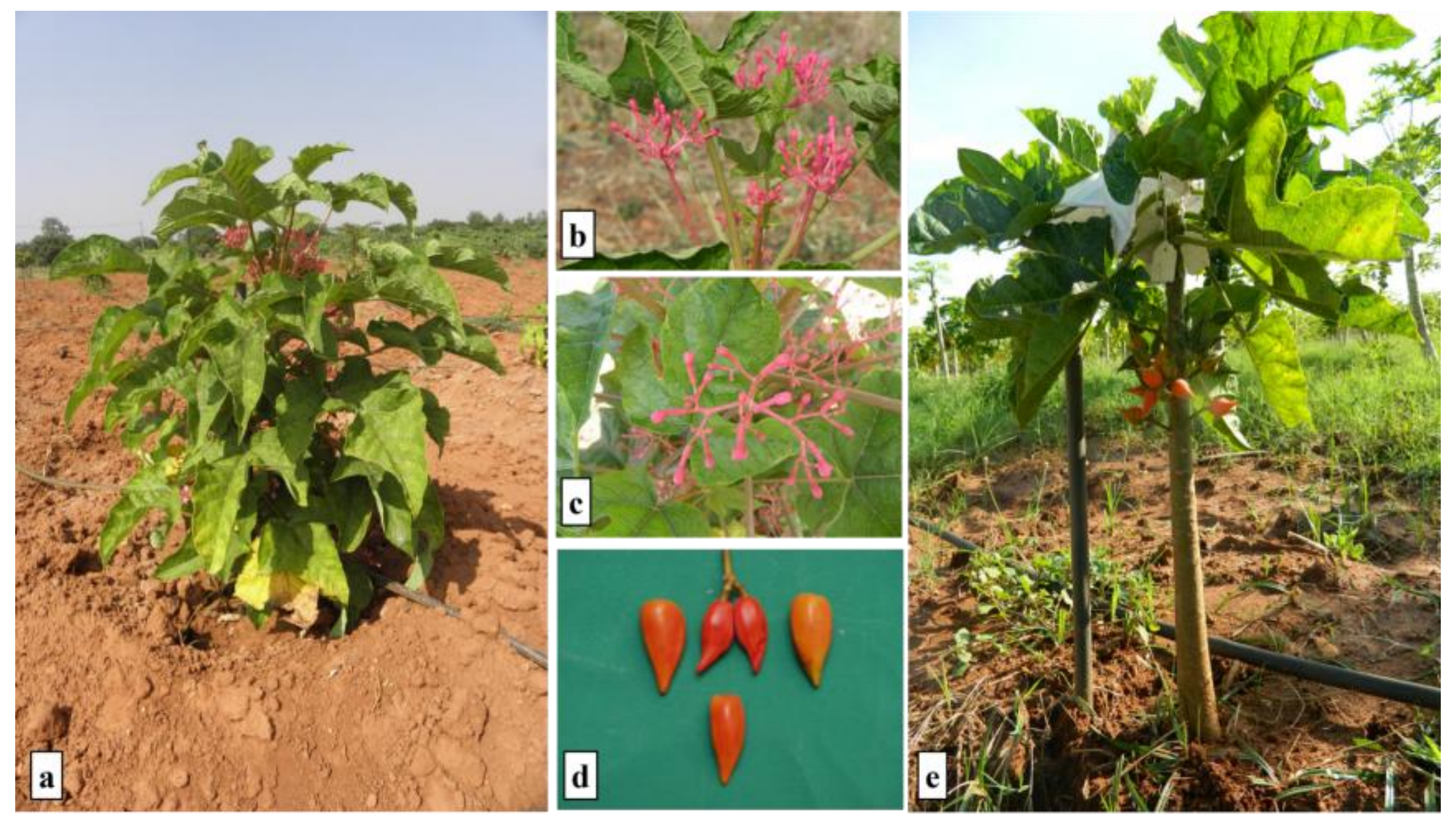


Plate.7 Field view of female parents used in hybridization

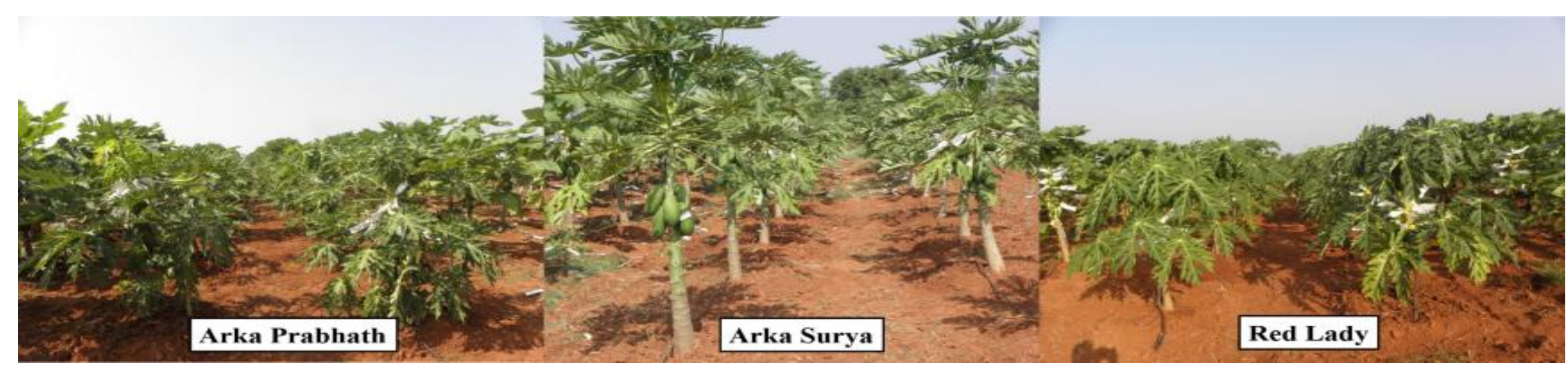

Plate.8 Steps involved in hybridization

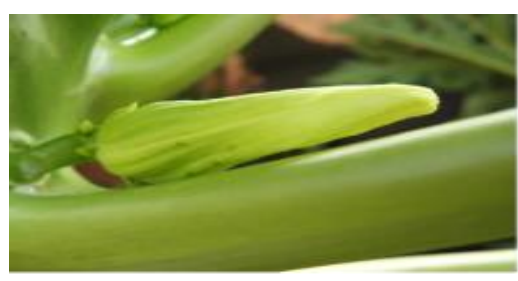

a) Unopened female flower

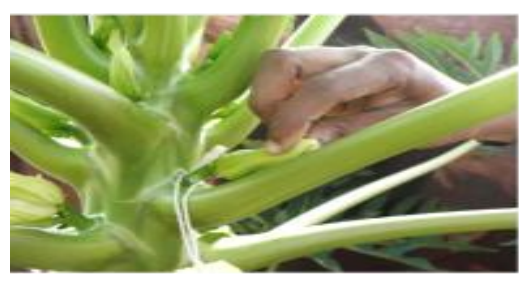

d) Forcing female flower to open

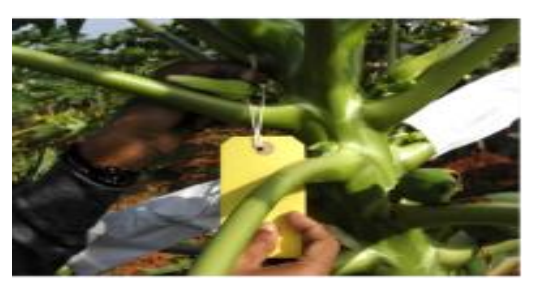

g) Labelling

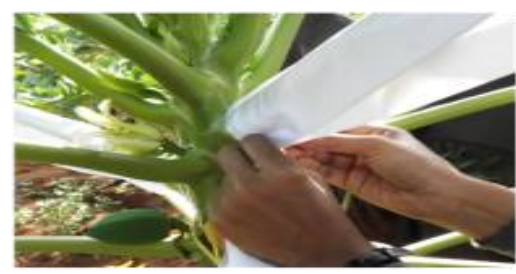

b) Bagging of female flower

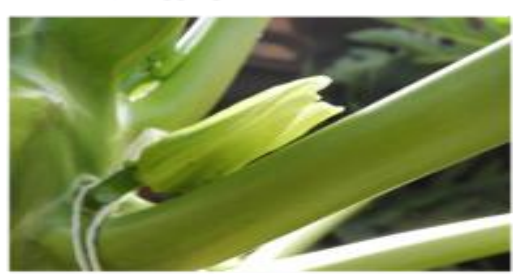

e) Opened female flower for crossing

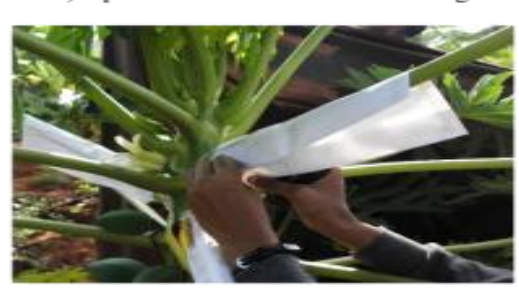
h) Covering crossed female flower with
Butter paper cover

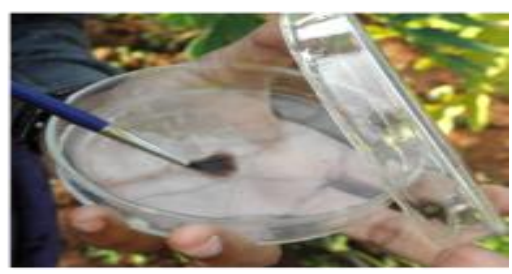

c) Preparation of pollen slurry

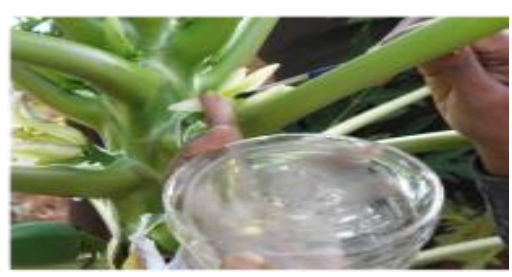

Smearing of pollen slurry on stigmatic

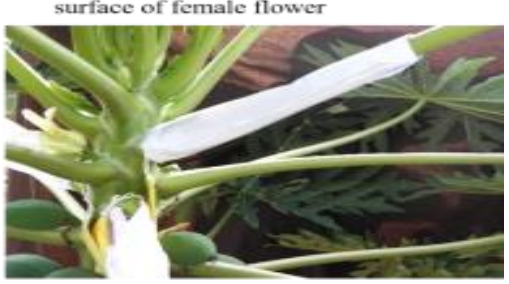

i) Crossed female flower 
Plate.9 Field view of fruit set recorded under various intergeneric crosses

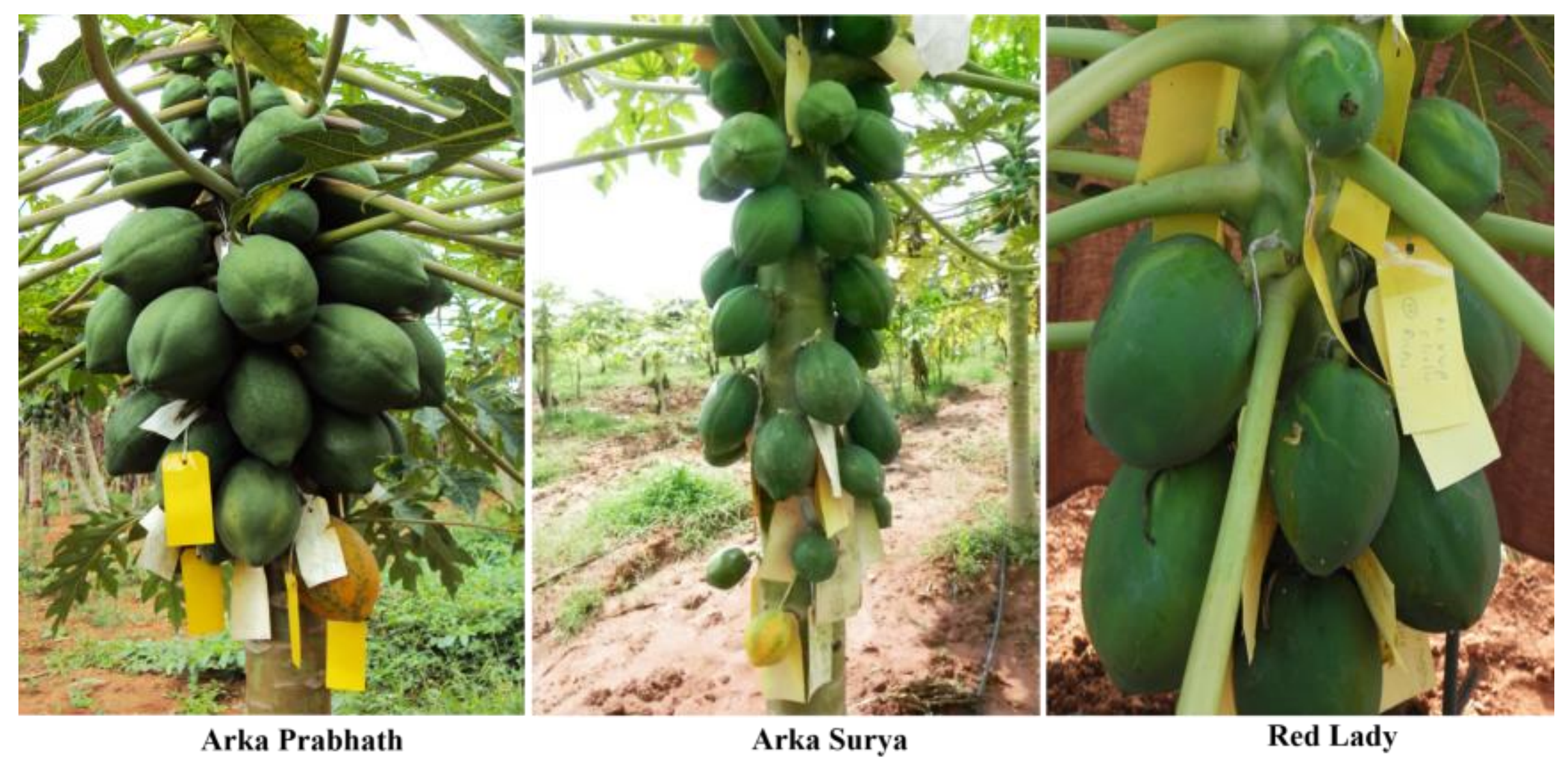


Plate.10 View of seed set recorded in Arka Prabhath across different male parents used for hybridization

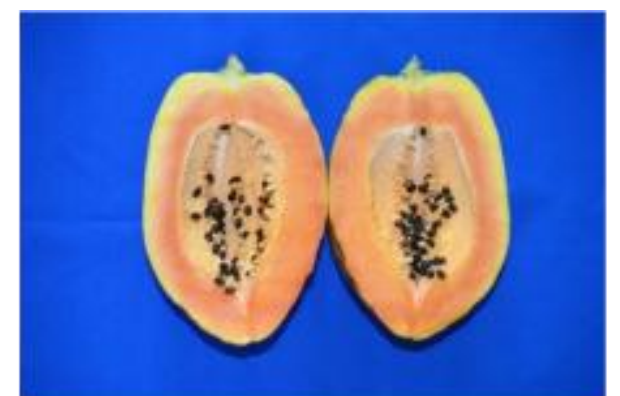

Arka Prabhath X $V$ cauliflora (Control)

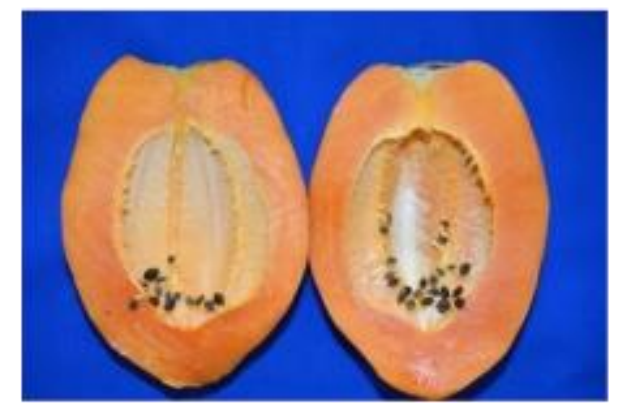

Arka Prabhath X $\boldsymbol{V}$ cundinamarcensis (Control) Arka Prabhath X $\boldsymbol{V}$ cundinamarcensis (Nutrient solution)

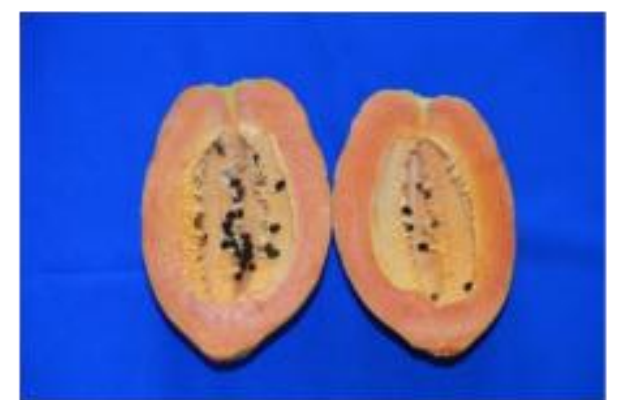

Arka Prabhath X $V$. parviflora (Control)

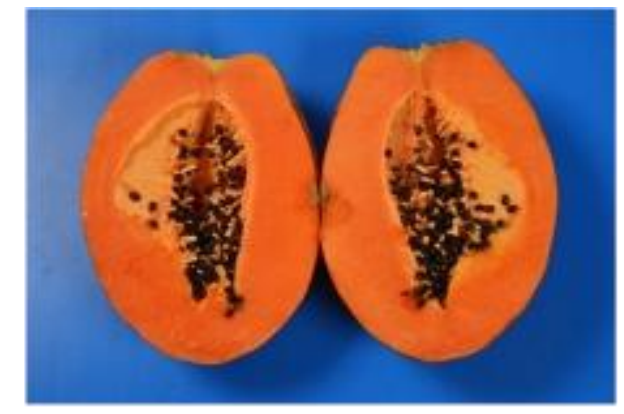

Arka Prabhath X $\boldsymbol{V}$ cauliflora (Nutrient solution)
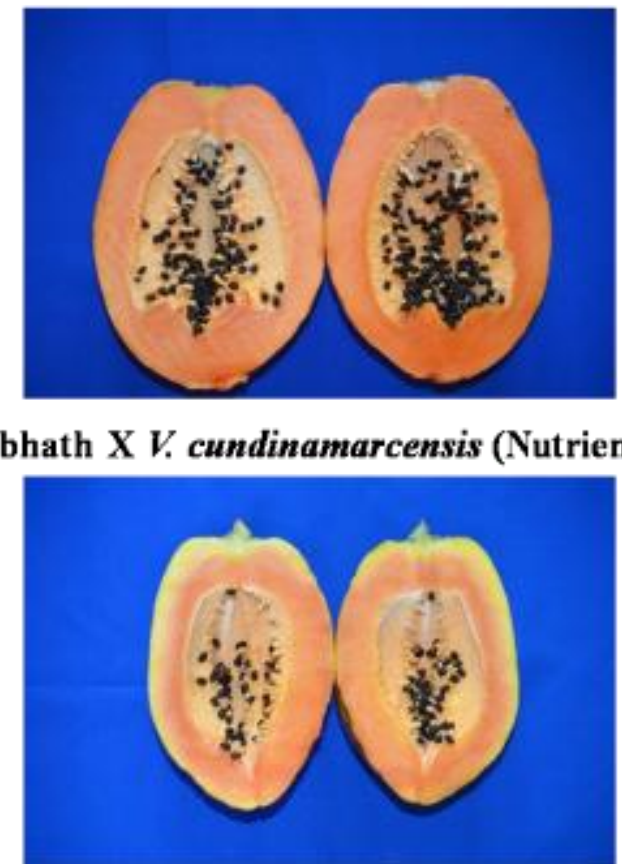

Arka Prabhath X V parviflora (Nutrient solution) 
Plate.11 View of seed set recorded in Arka Surya across different male parents used for hybridization

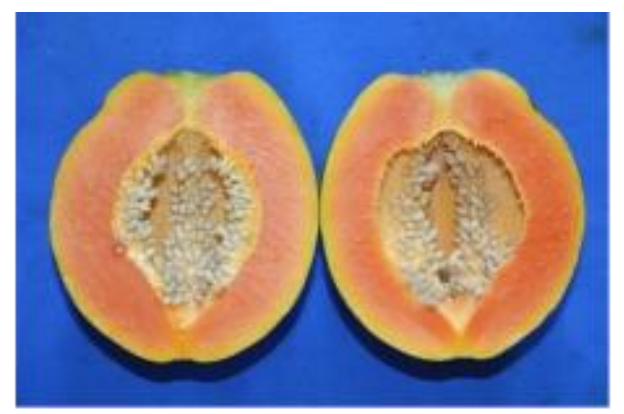

Arka Surya X V. cauliflora (Control)

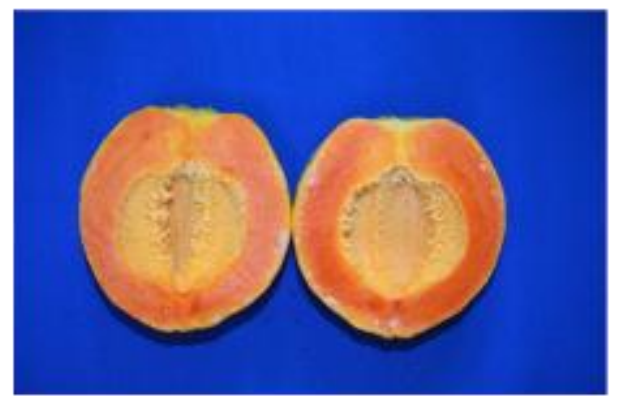

Arka Surya X V. cundinamarcensis (Control)

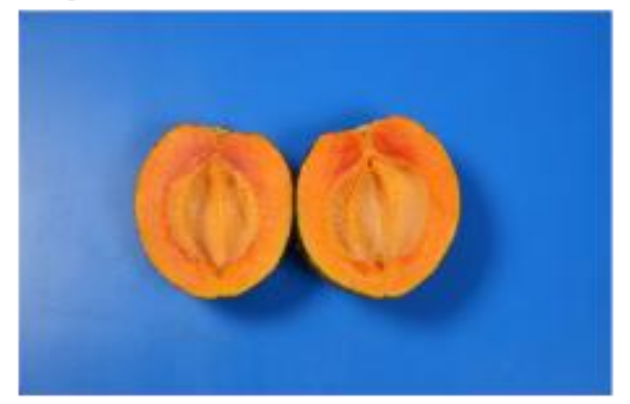

Arka Surya X V.parviflora (Control)

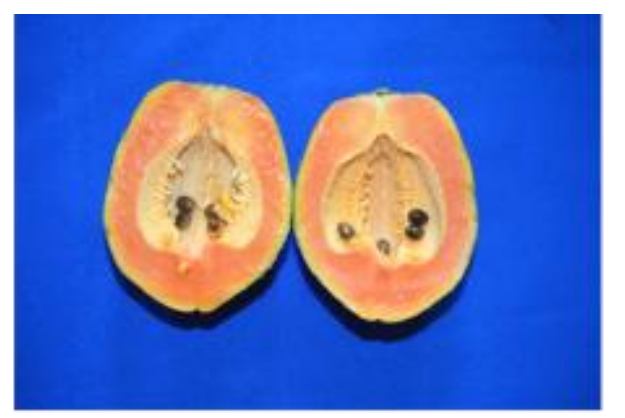

Arka Surya X V. cauliflora (Nutrient solution)

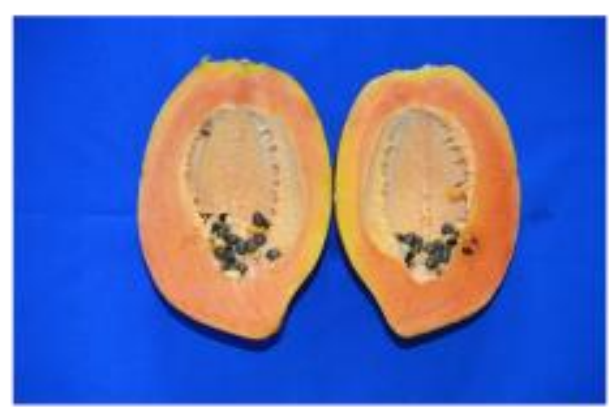

Arka Surya X V. cundinamarcensis (Nutrient solution)

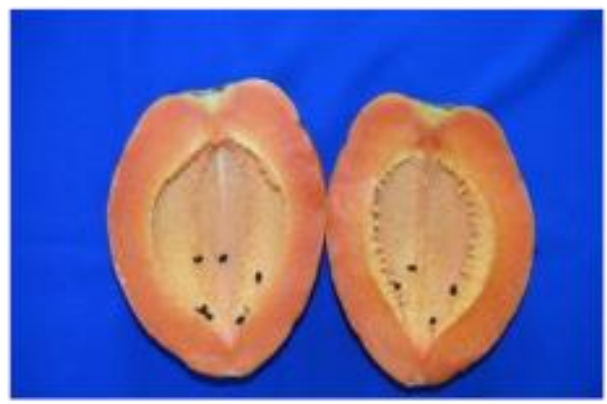

Arka Surya X V.parviflora (Nutrient solution) 
Plate.12 View of Seed set recorded in Red Lady across different male parents used for hybridization

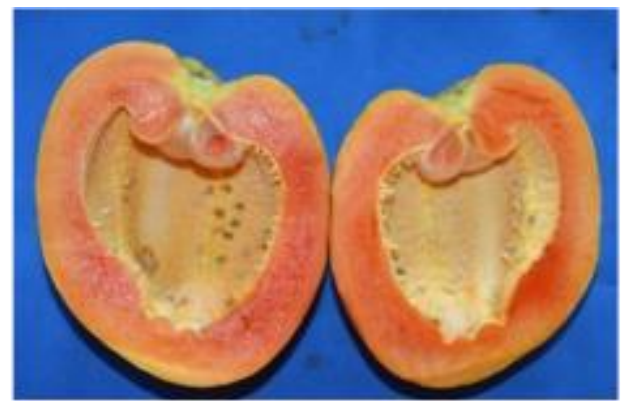

Red Lady X $V$. cauliflora (Control)

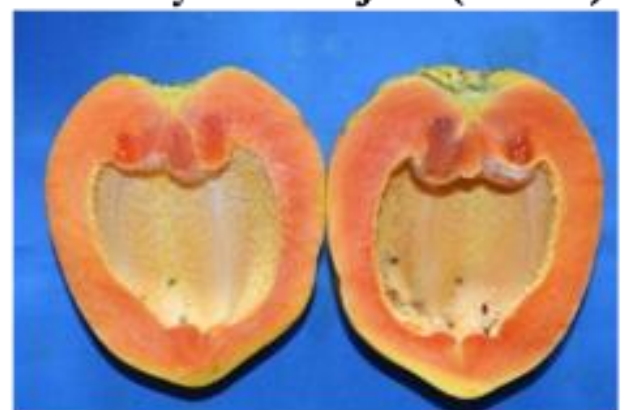

Red Lady X $\boldsymbol{V}$ cundinamarcensis (Control)

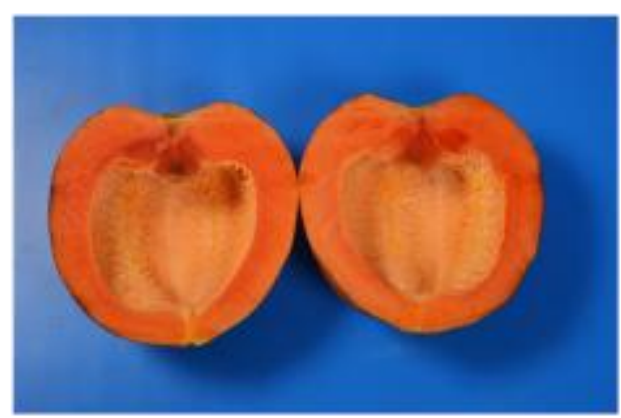

Red Lady X V.parviflora (Control)

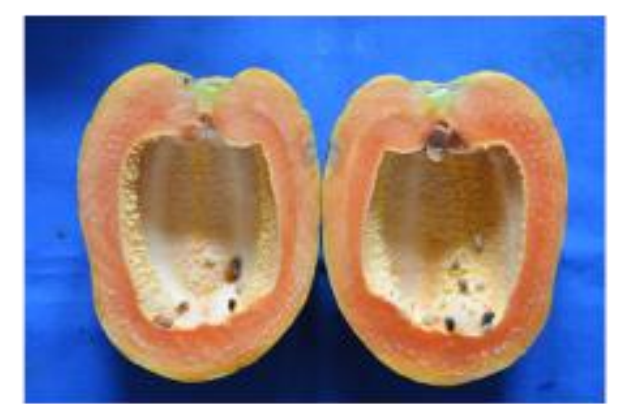

Red Lady X $V$. cauliflora (Nutrient solution)

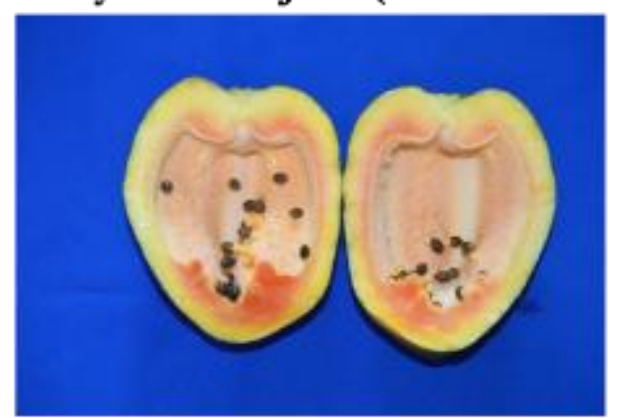

Red Lady X V cundinamarcensis (Nutrient solution)

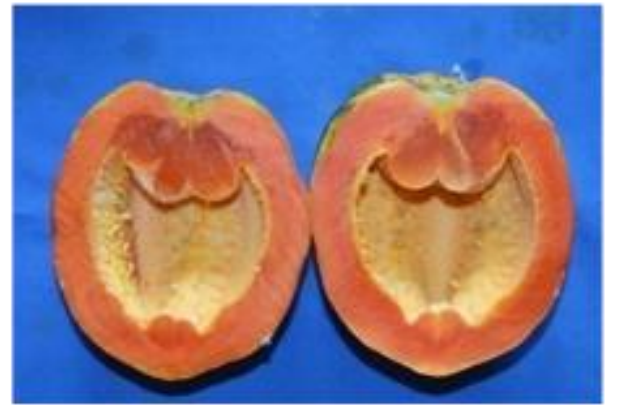

Red Lady X $\boldsymbol{V}$. parviflora (Nutrient solution) 
In the present study, as expected, good germination $(82.29,50.00$ and $30.00 \%$ in Red lady crosses) was recorded in the seeds obtained using nutrient solution for crossing, which is attributed to the proper development of embryo with good bold seeds having sufficient food reserve for seedling development. Similar results were obtained by earlier workers (Thirugnanvel, 2010). In control treatments due to lack of food reserve (endosperm) improper growth and development of embryo was observed resulting in poor germination.

\section{Yield of crossed fruits (kg/tree)}

The data on yield of crossed fruits per tree recorded in the intergeneric crosses (Table 4) revealed significant differences, which was in the range of 2.50 to $48.50 \mathrm{~kg} /$ tree. Highest yield was recorded in cross involving Arka Prabhath $\times \quad V$. cauliflora $\left(\mathrm{T}_{2}, 48.50 \mathrm{~kg} / \mathrm{tree}\right)$ followed by Arka Prabhath $\mathrm{x} V$. cundinamarcensis $\left(\mathrm{T}_{4}, 40.00 \mathrm{~kg} /\right.$ tree) and Arka Prabhath $\mathrm{x} V$. parviflora $\left(\mathrm{T}_{6}, 38.00\right.$ $\mathrm{kg} /$ tree). The lower yield was recorded in cross involving Arka Surya x V. parviflora $\left(\mathrm{T}_{11}, 2.50 \mathrm{~kg} / \mathrm{tree}\right)$ which was on par with Arka Surya x Vascnoncellea cauliflora $\left(\mathrm{T}_{7}\right.$, $3.00 \mathrm{~kg} /$ tree), Red Lady x V. parviflora $\left(\mathrm{T}_{17}\right.$, $3.50 \mathrm{~kg} /$ tree) and Arka Surya $\mathrm{x} V$. cundinamarcensis $\left(\mathrm{T}_{9}, 4.00 \mathrm{~kg} /\right.$ tree $)$.

In the present study, as expected, all the cross combinations which used nutrient solution for crossing, had resulted in higher fruit recovery (48.50, 40.00 and $38.00 \mathrm{~kg}$ in Arka Prabhath crosses) as compared to control treatments. As yield is a component of several factors involving the genotypes, its cross compatibility, initial fruit set, development and final recovery. The combination involving Arka Prabhath as female parent has resulted in higher yield per tree $(48.50,40.00$ and $38.00 \mathrm{~kg}$ ) as compared to other two female parents, indicating its ability to be the best combiner for intergeneric cross with the Vasconcellea species used in the present investigation.

In conclusion, intergeneric hybridization was carried out to know the cross compatibility between papaya varieties (Arka Prabhath, Arka Surya and Red Lady) with Vasconcellea species (V. cauliflora, $V$. cundinamarcensis and $V$. parviflora). Arka Prabhath was found to be a good combiner with all the three wild species ( $V$. cauliflora, $V$. cundinamarcensis and $V$. parviflora). Among male parents, Vasconcellea. cauliflora was found to be good combiner with all three female parents (Arka Prabhath, Arka Surya and Red Lady). Hence Arka Prabhath with V. cauliflora cross combination was found to be good for intergeneric hybridization which has resulted in more mean number of fertile seeds (heterotic combinations) which ultimately helps in generating more number of intergeneric population for screening of PRSV.

\section{References}

Arumuganathan, K. and Earle, E. D., 1991, Nuclear DNA content of some important plant species. Plant Molecular Biology Reporter, 9: 208218.

Azad, M. A. K, Rabbani, G. and Amin, L., 2012, Plant regeneration and somatic embryogenesis from immature embryos derived through interspecific hybridization among different Carica Species. Int. J. Mol. Sci., 13: 1706517076.

Badillo, V. M., 2000, Carica L. vs Vasconcellea St. Hill. (Caricaceae): con la rehabilitacion de este ultimo. Ernstia 10: 74-79.

Bennet, M. D. and Leitch, I. J., 2005, Plant DNA C-values database.

Bharathi, N., 2011, Intergeneric crossing, 
intervarietal progeny evaluation and mutagenic studies in papaya (Carica papaya L.), M. Sc. Thesis, UAS Bengaluru.

Chan, Y. K., 2004, Breeding papaya for resistance to ringspot virus disease in Malaysia. Acta Hort. 632: 57-64.

Chandra, J. K. and Samuel, L. D. K., 1999, Viral and phytoplasmal diseases of papaya in India. Indus publishing Co., New Delhi, 724.

Conover, R. A. and Litz, R. E., 1981, Tolerance to papaya ringspot virus in papaya (Carica papaya L.) Phytopathology, 71: 868.

Dinesh, M. R., Rekha, A., Ravishankar, K. V., Praveen, K. S. and Santosh, L. C., 2007, Breaking the intergeneric crossing barrier in papaya using sucrose treatment. Scientia Hort., 114: 33-36.

Gonsalves D, 1998. Control of Papaya ringspot virus: a case of study. Annual Review of Phytopathology 36: 415-37.

Gonsalves, D. C., Gonsalves, S., Ferreira, K., Pitz, M., Fitch, R. M. and Slinghtom, J., 2004, Transgenic virus resistant papaya: from hope to reality for controlling of papaya ring spot virus in Hawaii,: 1-12.

Gonsalves, D., 1994, Papaya Ring Spot. In: Compendium of Tropical Fruit Diseases. (Ploetz, R. C., ed.). MN, USA: APS Press. Pp. 67.

Hussain, S. and Varma, A. A., 1994, Occurrence of papaya ring spot virus from Amritsar (Punjab) India. $J$. Phytopath. Res., 7: 77-78.

Jayavalli, R., Balamohana, T. N., Manivannan, N. and Govindaraj, M., 2011. Breaking the intergeneric hybridization barrier in Carica papaya and Vasconcellea cauliflora, Scientia Hort., 130: 787-794.

Jimenez, H. and Horovitz, S., 1958, interspecific os intergeneric o sen Caricacea. Sysus implacaciones fitotecnicas CJA Venezuela. Agron.
Trop. 21: 123-143.

Lichamo, J. Y., 2015, Evaluation of F6 Intergeneric population of papaya (Carica papaya L) for resistance to papaya ring spot virus (PRSV). M. Sc. Thesis, University of horticultural sciences, Baglkot.

Litz, R. E., 1984, Papaya, Handbook of plant cell culture, 2:349-368.

Magdalita, P. M., Drew, R. A., Adkins, S. W., and Godwin, I. D., 1997 Morphological, molecular and cytological analysis of Carica papaya $\mathrm{x}$ C. cauliflora interspecific hybrids. Theor. Appl. Genet., 95: 224-229.

Manshardt, R. M. and Wenslaff, T. F., 1989, Zygotic polyembryony in interspecific hybrids of Carica papaya and Carica cauliflora. J. Amer. Soc. Hort. Sci., 114(4): 684-689.

Manshardt, R. M., Zee, F., Ferreira, S., Luis, S., Sondur, S., Wenslaff, T., Fitch, M. Gonsalves, D., Slightom, J. L., Sanford, J. and Stiles, J., 1995, Papaya breeding for PRSV resistance. International Symposium on Tropical Fruits. Acta Hort., 370: 232.

Mekako, H. V. and Nakasone, H. Y., 1975, Intergeneric hybridization among different Carica species. J. Amer. Soc. Hort. Sci., 100: 14-19.

Muthulakshmi, S., Balamohan, T. N., Amutha, R., Babyrani, W., Indira, K. and Mareeswari, P., 2007, Inter specific hybridization in papaya (Carica papaya L.). Res. J. Agric. Biological Sci., 3(4): 260-263.

Naksone, H. K. and Paull, R. E., 1998, Tropical fruits, $\mathrm{CAB}$ international, Wallingford, 132-148.

Purcifull D. E., Edwardson, J. R., Hiebert, E. and Gonsalves, D., 1984, Papaya ringspot virus CMI/AAB Descriptions of Plant Viruses, No. 292. (CAB International, Wallingford, UK)

Shukla, D. D., Ward, C. W. and Brunt, A. A., 
1994, The potyviridae I eds., Wallingford, CAB Internation, UK.

Singh, R. N., 1964, Papaya breeding- A review, Indian J. Hort. 21: 148-154.

Storey, W. B., 1938, Segregation of sex types in solo papaya and their application to the selection of seed. American society for Hortil. Sci. proceedings, 35: 83-85.

Sundarraj, N., Nagaraju, S., Vekatarama, H. N. and Jagannatha, M. K., 1972, Design and analysis of field experiments. UAS
Publication, Bangalore.

Thirugnanavel, A., 2010, Breeding for PRSV resistance in papaya (Carica papaya $\mathrm{L}$.) through germplasm screening and intergeneric hybridization. Ph. D. Thesis, Tamil Nadu Agricultural University Coimbatore.

Zee, F., 1985, Breeding of papaya virus tolerance in solo papayas, Carica papaya $\mathrm{L}$. $\mathrm{Ph}$. D. Thesis, University of Honolulu, Pp. 105.

\section{How to cite this article:}

Deepa U. Pujar, C. Vasugi, D. Adiga, M.K. Honnabyraiah, H.S. Vageeshbabu, J. Jayappa and Kanupriya. Study on Cross Compatibility of Intergeneric Hybridization between Carica with Vasconcellea Species. Int.J.Curr.Microbiol.App.Sci. 8(04): 1514-1537. doi: https://doi.org/10.20546/ijcmas.2019.804.177 